2014-3

\title{
Aircraft Route Optimization for Formation Flight
}

Jia Xu

Stanford University

Andrew Ning

Brigham Young University - Provo, aning@byu.edu

Geoffrey Bower

Stanford University

Ilan Kroo

Stanford University

Follow this and additional works at: https://scholarsarchive.byu.edu/facpub

Part of the Mechanical Engineering Commons

\section{Original Publication Citation}

Xu, J., Ning, A., Bower, G., and Kroo, I., "Aircraft Route Optimization for Formation Flight," Journal of Aircraft, Vol. 51, No. 2, Mar. 2014, pp. 490-501. doi:10.2514/1.C032154

\section{BYU ScholarsArchive Citation}

Xu, Jia; Ning, Andrew; Bower, Geoffrey; and Kroo, Ilan, "Aircraft Route Optimization for Formation Flight" (2014). Faculty Publications. 1675.

https://scholarsarchive.byu.edu/facpub/1675

This Peer-Reviewed Article is brought to you for free and open access by BYU ScholarsArchive. It has been accepted for inclusion in Faculty Publications by an authorized administrator of BYU ScholarsArchive. For more information, please contact ellen_amatangelo@byu.edu. 


\title{
Aircraft Route Optimization for Formation Flight
}

\author{
Jia Xu*, S. Andrew Ning*, Geoffrey Bower* \\ and Ilan $\mathrm{Kroo}^{\dagger}$ \\ Stanford University, Stanford, CA, 94305, U.S.A
}

\begin{abstract}
We quantify the fuel and cost benefits of applying extended formation flight to commercial airline operations. Central to this study is the development of a bi-level, mixed integer-real formation flight optimization framework. The framework has two main components: 1) a continuous domain aircraft mission performance optimization and 2) an integer optimization component that selects the best combination of optimized missions to form a formation flight schedule. The mission performance reflects the effects of rolled-up wakes, formation heterogeneity, and formation-induced compressibility. The results show that an airline can use formation flight to reduce fuel burn by $5.8 \%$ or direct operating cost by $2.0 \%$ in a long-haul international schedule. The savings increase to $7.7 \%$ in fuel or $2.6 \%$ in cost for a large-scale, transatlantic airline alliance schedule. These results include the effects of a conservative fuel reserve for formation flight. Sensitivity studies show that a modest reduction in the cruise Mach number may be sufficient to manage the impact of formation-induced compressibility effects on system-level formation flight performance. We demonstrate that the potential savings from extended formation flight-an operational improvement using existing aircraft - can approach those claimed for advanced vehicle technologies and unconventional configurations.
\end{abstract}

\section{Nomenclature}

$\begin{array}{ll}e & \text { Vector of 1's } \\ \Delta x & \text { Longitudinal separation between incoming vortex and nearest wingtip } \\ \Delta y & \text { Lateral separation between incoming vortex and nearest wingtip } \\ \Delta z & \text { Vertical separation between aircraft in formation } \\ \Delta C_{D i} & \text { Change in aircraft induced drag due to formation flight } \\ \Delta t_{a} & \text { Change in arrival time } \\ \Delta t_{a \max } & \text { Maximum allowable change in arrival time } \\ \Delta t_{d} & \text { Change in departure time } \\ \Delta t_{d \text { max }} & \text { Maximum allowable change in departure time } \\ \boldsymbol{J}_{\text {missionk }} & \text { Optimal fuel burn or cost for each solo or formation mission } \\ J_{\text {schedule }} & \text { Schedule optimization objective function } \\ l_{a t} & \text { Latitude for flight state } k \\ l_{k} n_{k} & \text { Longitude for flight state } k \\ T S F C & \text { Thrust specific fuel consumption } \\ b & \text { Wing span } \\ b_{0} & \text { Initial spacing between a vortex pair } \\ c_{b l k} & \text { DOC components that scale with the block time } \\ c_{f l t} & \text { DOC components that scale with the flight time } \\ c_{l a b o r} & \text { Maintenance labor cost } \\ c_{\text {oil }} & \text { Lubrication oil cost }\end{array}$

*Ph.D. Department of Aeronautics \& Astronautics. Member AIAA.

${ }^{\dagger}$ Professor, Department of Aeronautics \& Astronautics. Fellow AIAA. 


\begin{tabular}{|c|c|}
\hline$C_{L}$ & Aircraft lift coefficient \\
\hline$d_{i}$ & Great circle distance between the arrival and departure airports of aircraft $i$ \\
\hline$h_{k}$ & Altitude for flight state $k$ \\
\hline$i_{\text {fuel }}$ & Formation fuel burn rate index \\
\hline$J_{c}$ & Cost objective \\
\hline$J_{f}$ & Fuel burn objective \\
\hline$k_{\text {inflate }}$ & Inflation factor \\
\hline$\dot{m}_{f}$ & Formation fuel burn rate \\
\hline$M_{k}$ & Mach number for flight state $k$ \\
\hline$n_{\text {cabin }}$ & Number of cabin crew \\
\hline$n_{\text {cockpit }}$ & Number of cockpit crew \\
\hline$n_{a}$ & Number of aircraft in a formation \\
\hline$q_{\infty}$ & Freestream dynamic pressure \\
\hline$r$ & Radial position from vortex core \\
\hline$r_{k}$ & Aircraft range over segment $k$ \\
\hline$T / D$ & Thrust-to-drag \\
\hline$T_{0}$ & Sea level static thrust \\
\hline$t_{b l k}$ & Block time \\
\hline$t_{f l t}$ & Flight time \\
\hline$t_{a}$ & Scheduled arrival time \\
\hline$t_{k}$ & Time of flight state $k$ \\
\hline$U_{\infty}$ & Freestream velocity \\
\hline$V_{\theta}$ & Tangential velocity \\
\hline$V_{n}$ & Normalwash \\
\hline$W_{\text {airframe }}$ & Airframe weight \\
\hline$W_{\text {engines }}$ & Dry engine weight \\
\hline$W_{f}$ & Fuel burn \\
\hline$W_{k}$ & Weight for flight state $k$ \\
\hline$(T / D)_{\text {solo }}$ & Thrust-to-drag in solo operations \\
\hline $\boldsymbol{A}_{\text {schedulek }}$ & Binary matrix indicating which aircraft is in which formation \\
\hline $\boldsymbol{x}_{\text {schedule }}$ & Binary variable indicating whether or not a solo or formation mission is flown \\
\hline$d_{a i j}$ & Great circle distance between the arrival airports of aircraft $i$ and $j$ \\
\hline$d_{d i j}$ & Great circle distance between the departure airports of aircraft $i$ and $j$ \\
\hline$n_{m k}$ & Number of optimized candidate missions for formation size $k$ \\
\hline$r_{k \text { solo }}$ & Aircraft range over segment $k$ in solo operations \\
\hline$t_{a i}$ & Arrival time for aircraft $i$ \\
\hline$t_{d i}$ & Departure time for aircraft $i$ \\
\hline$W_{f_{c}}$ & Climb fuel burn \\
\hline$A R$ & Aspect ratio \\
\hline$D O C$ & Direct Operating Cost \\
\hline$S_{r e f}$ & Wing reference area \\
\hline$W_{M T O W}$ & Maximum takeoff weight \\
\hline \multicolumn{2}{|l|}{ Symbols } \\
\hline$\Delta \phi$ & Arrival or departure azimuth difference (minor angle) \\
\hline$\Gamma$ & Circulation \\
\hline$\phi_{a}$ & Arrival azimuth \\
\hline$\phi_{d}$ & Departure azimuth \\
\hline$\rho_{\infty}$ & Freestream air density \\
\hline$\kappa_{d}$ & Formation "aspect ratio" \\
\hline$\kappa_{t}$ & Formation flight time overlap coefficient \\
\hline
\end{tabular}

\section{Introduction}

Numerous studies have examined the aerodynamic benefits of close formation flight using both numerical and experimental means. ${ }^{1-9}$ These studies agree that formation flight has the potential to significantly reduce 
aircraft induced drag and fuel burn. The fuel savings from formation flight compare favorably with natural laminar flow wings, blended wing body configurations, and open-rotor engines. But unlike advanced vehicle technologies, formation flight can make use of existing aircraft with minimal modifications.

Our recent work examines the concept of extended formation flight, ${ }^{10}$ where aircraft are separated by streamwise spacing of 5-40 wingspans. The extended spacing may render formation flight safer and more compatible with commercial and cargo operations. The concept is the subject of a NASA experiment using C-17 transports. 9

In this paper we extend the bi-level optimization of Bower to better place extended formation flight in the context of real world airline operations $\frac{11] 12}{12}$ We extend the design framework with a cost model to address the economic and operational viability of formation flight. The analysis also incorporates, for the first time, a heterogeneous aircraft formation drag model based on a rolled-up wake 10 This allows large airline and airline alliance schedules, which are often flown by a mix of aircraft types, to be analyzed. The improved mission optimization tool also operates on the full 4-D trajectory of aircraft. Finally, we incorporate recent Euler CFD analysis of wake propagation to examine the impact of compressibility constraints on formation flight performance. $\frac{13}{13}$

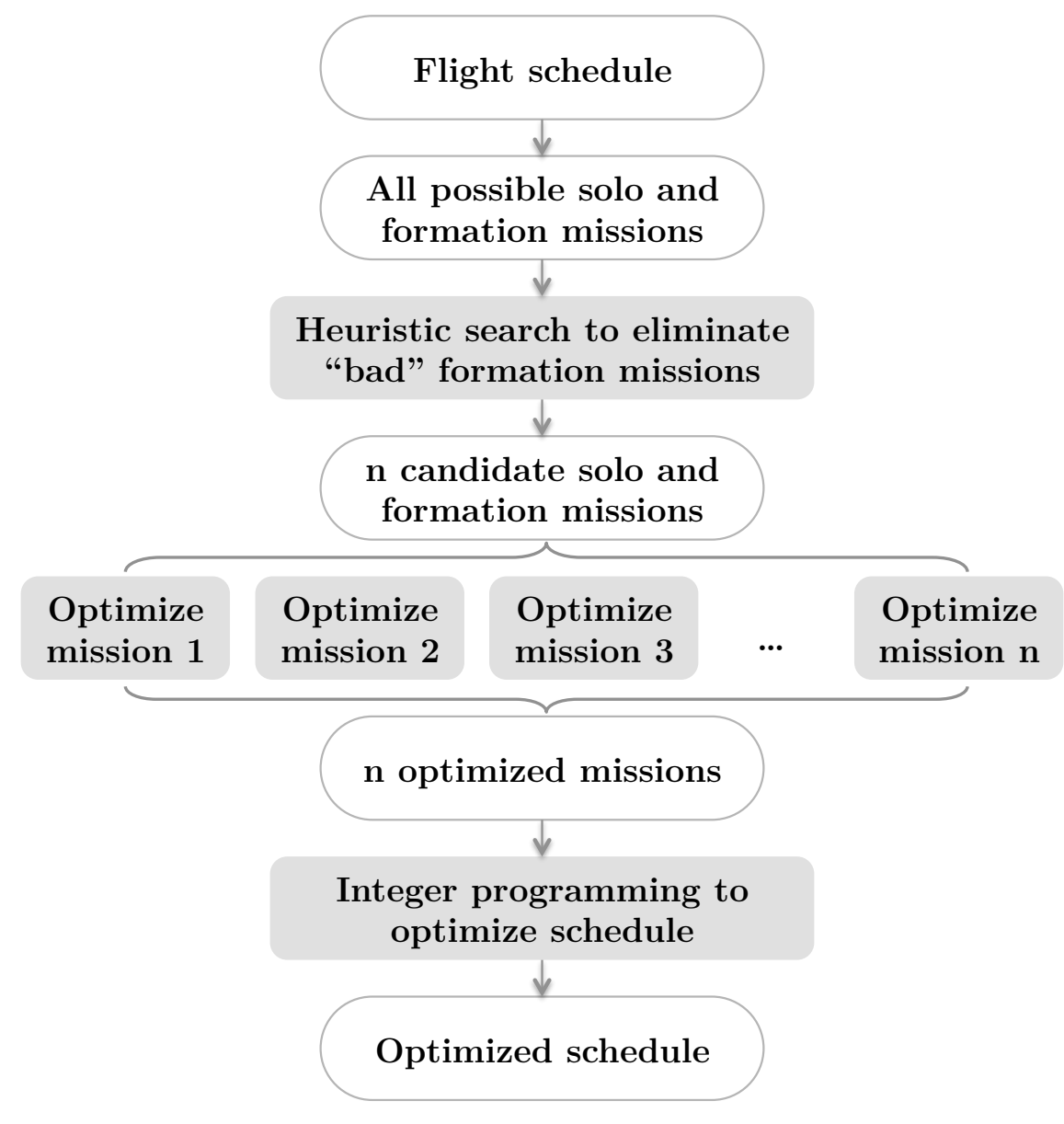

Figure 1. The architecture of the mission and flight schedule optimization.

Figure 1 illustrates the information flow of the optimization framework. The following definitions for route, mission and schedule apply:

- A route is defined by an origin-destination pair. Multiple missions can serve the same route.

- A mission can be flown by a single or a formation of aircraft. Each aircraft in the formation serves one route. The mission optimization operates on the 4-D trajectory of all of the aircraft.

- A schedule is the set of all missions to be flown. The schedule optimization determines which mission (out of the set of all possible missions) should be flown.

The formation flight optimization framework uniquely combines the integer programing methods typically associated with fleet scheduling problems $\sqrt{14}\left[\frac{16}{16}\right.$ with continuous domain aircraft performance optimization. 
The input to optimization is an airline flight schedule. The size of the problem grows rapidly with the number of scheduled flights. To deal with this growth we apply the heuristic search method described in Section III to identify candidate formation missions that are likely to benefit from formation flight. These candidate missions are then optimized for minimum fuel burn or cost using efficient, gradient-based optimization. The mission optimization operates on the Mach number, altitude, longitude, and latitude of the aircraft in solo and formation segments. The design variables can also include the departure and arrival time of individual flights to provide additional scheduling flexibility.

The next step is to find the best flight schedule amongst all possible combinations of candidate missions. We pose the schedule optimization as an integer-programming problem and solve it using branch and bound type algorithms. The binary design variables define which individually optimal formation and solo missions should be flown.

\section{Formation Aerodynamics}

The drag reduction mechanism in formation flight is relatively well understood. Figure 2 shows that as an aircraft flies through the air it leaves behind regions of downwash inboard and upwash outboard of its wings. A trailing aircraft can fly through the upwash to reduce its induced drag at fixed lift. In the case of extended formation flight the downstream aircraft exert essentially no influence on the lead aircraft. The great longitudinal separation also means that the evolution of the wake becomes an important consideration in the assessment of formation drag savings.

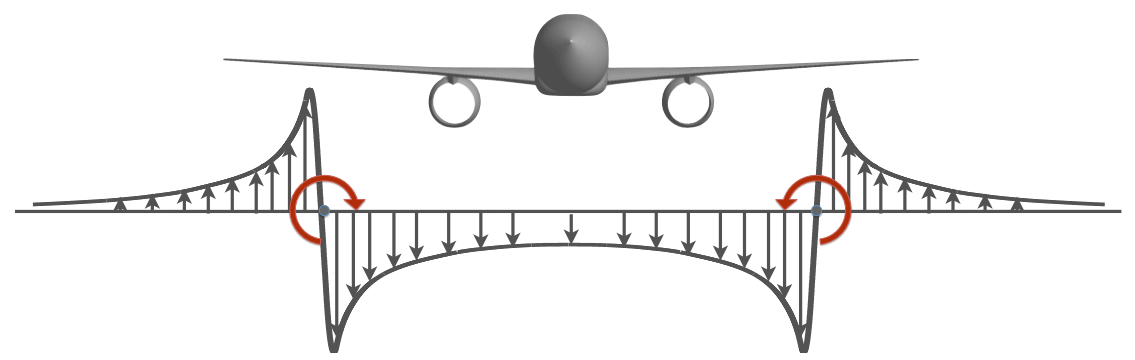

Figure 2. The drag reduction mechanism behind formation flight: the outboard wake upwash from a leading aircraft.

Ning10 describes the aircraft wake development model for extended formation flight. This method uses a far-field conservation method ${ }^{17}$ to compute the rolled-up vorticity distribution of an aircraft's wake. We augment this model with experimental data on vortex core sizes $\frac{18}{18}$ and a viscous decay model based on large eddy simulations and experimental data ${ }^{19}$ This augmented Betz method for agrees well with Navier-Stokes

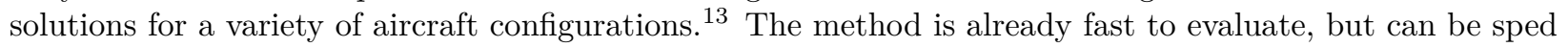
up further for this application. King and Gopalarathnam ${ }^{20}$ have shown that a formation of elliptically loaded aircraft has very nearly the same induced drag as one that is optimally loaded when subject to trim constraints (for planar wings with no overlap in the wing traces). Thus, to a good approximation, we can assume that each aircraft in the formation is elliptically loaded. The tangential velocity profile induced by the wake vortices can now be pre-computed (properly normalized). Figure 3 shows an example of this self-similar velocity profile. This example is computed using the augmented Betz method, but any reasonable method (Navier-Stokes calculation or experimental data) could be used.

For an elliptically loaded wing, the spacing between the rolled-up vortices is given by

$$
b_{0}=\frac{\pi}{4} b
$$

and the total vortex circulation by

$$
\Gamma_{0}=\frac{U_{\infty} C_{L} S_{r e f}}{2 b_{0}}
$$

Using these parameters and the non-dimensional tangential velocity profile of the rolled-up vortex, we can compute the vortex-induced velocity on a trailing aircraft. The wake is also allowed to decay to account for differences in longitudinal separation. For the relatively moderate separation distances in the present study 


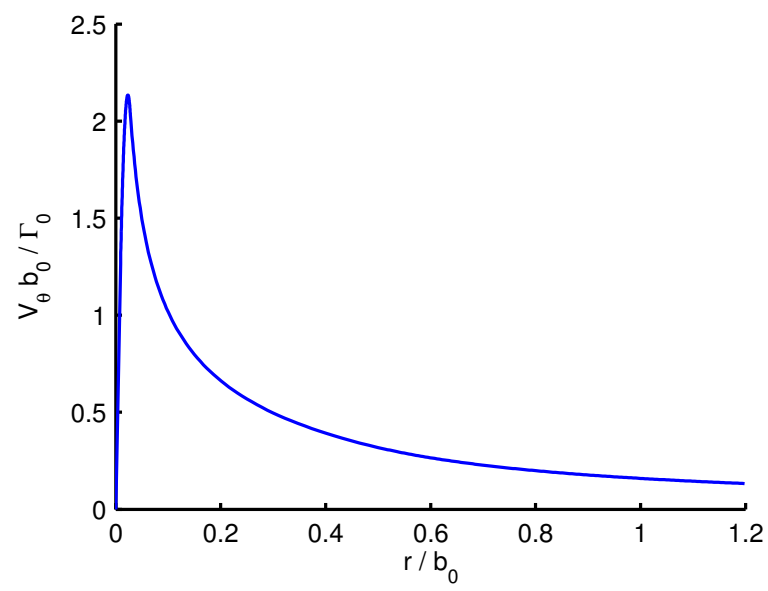

Figure 3. The tangential velocity distribution of a wake vortex as a function of distance from the vortex center (elliptically loaded wing).

(less than 40 spans) we use the diffusion phase of Holzäpfel's model ${ }^{19}$ to characterize wake decay:

$$
\Gamma\left(t^{*}\right)=\Gamma_{0}\left[A-\exp \left(\frac{-R^{* 2}}{\nu_{1}^{*}\left(t^{*}-T_{1}^{*}\right)}\right)\right]
$$

where $A, R^{*}, T_{1}^{*}, \nu_{1}^{*}$ are coefficients tuned using large eddy simulations and $t^{*}=\Gamma_{0} /\left(2 \pi b_{0}^{2}\right) t$. The induced drag of the trailing aircraft is then given by

$$
D_{i}=\frac{C_{L}^{2}}{\pi A R} q_{\infty} S_{r e f}+\int \rho_{\infty} V_{n} \Gamma d s
$$

Where the integral is along the wing trace, and the normalwash is due only to the wake influence from upstream aircraft.

For heterogeneous formations in which the aircraft differ in size and/or engine efficiency, the aircraft ordering and formation arrangement affects the total formation fuel burn. In this study we consider the 2-aircraft echelon formation and the three 3-aircraft formation configurations shown in Figure 4 For heterogeneous formations this results in 2 possible arrangements for 2-aircraft formations, and 18 possible arrangements for 3-aircraft formations. Intuition suggests that the most efficient arrangement places heavier aircraft in the middle (closest approximation to an elliptical distribution of lift across the formation), and less fuel efficient aircraft in the back (where they can take advantage of reduced fuel burn rates). This is a useful rule of thumb, but as explored in more detail by Ning these guidelines do not always hold. 21

Of the two governing parameters in choosing the formation arrangement (relative TSFC and relative weight), the TSFC has a more dominant effect. Thus, when evaluating a 3-aircraft formation, rather than evaluate all 18 potential arrangements, we sort the aircraft streamwise by increasing TSFC. This now leaves three potential arrangements to evaluate (the three formation types shown in Figure 4). The total fuel burn of the formation is proportional to

$$
\dot{m}_{f} \propto \sum_{j} D_{j} T S F C_{j}
$$

Assuming that formation flight affects only the induced component of the drag, and because the aircraft in formation travel at the same dynamic pressure, the fuel burn rate between formations can be compared using the index

$$
i_{\text {fuel }}=\sum_{j}\left(C_{D i} S_{r e f}\right)_{j} T S F C_{j}
$$

Using the methodology discussed earlier, the induced drag coefficient of each aircraft can be estimated using the following functional form.

$$
C_{D i}=f\left(C_{L}, S_{r e f}, b, \Delta x, \Delta y, \Delta z, \text { formation type }\right)
$$


This calculation is repeated for all aircraft in the formation, and the formation fuel burn index calculation is repeated for all formation arrangements. Finally, the formation with the minimum fuel burn index is selected, and the corresponding induced drag for each aircraft in the formation is used in the performance analysis.

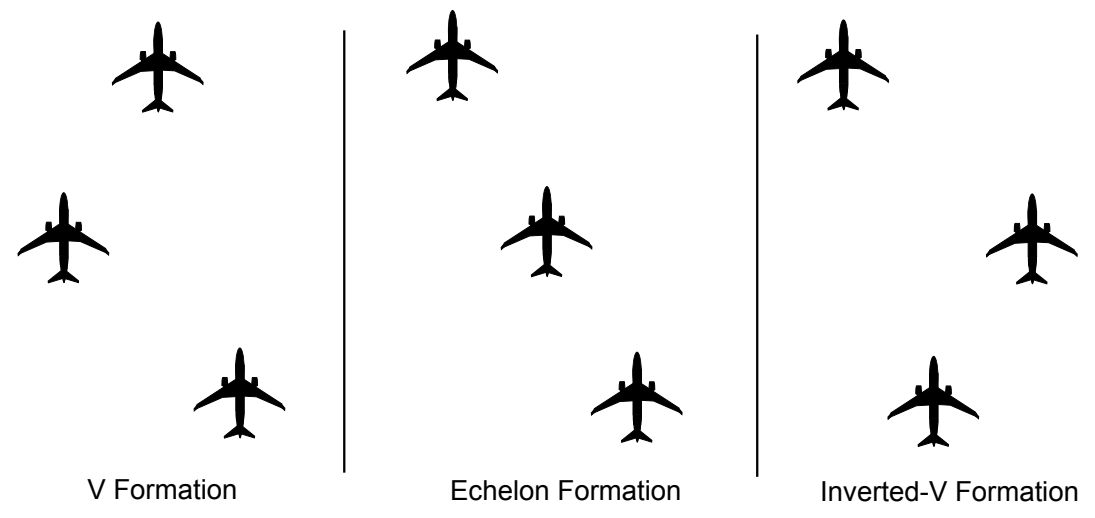

Figure 4. The three formation configurations included in the induced drag model.
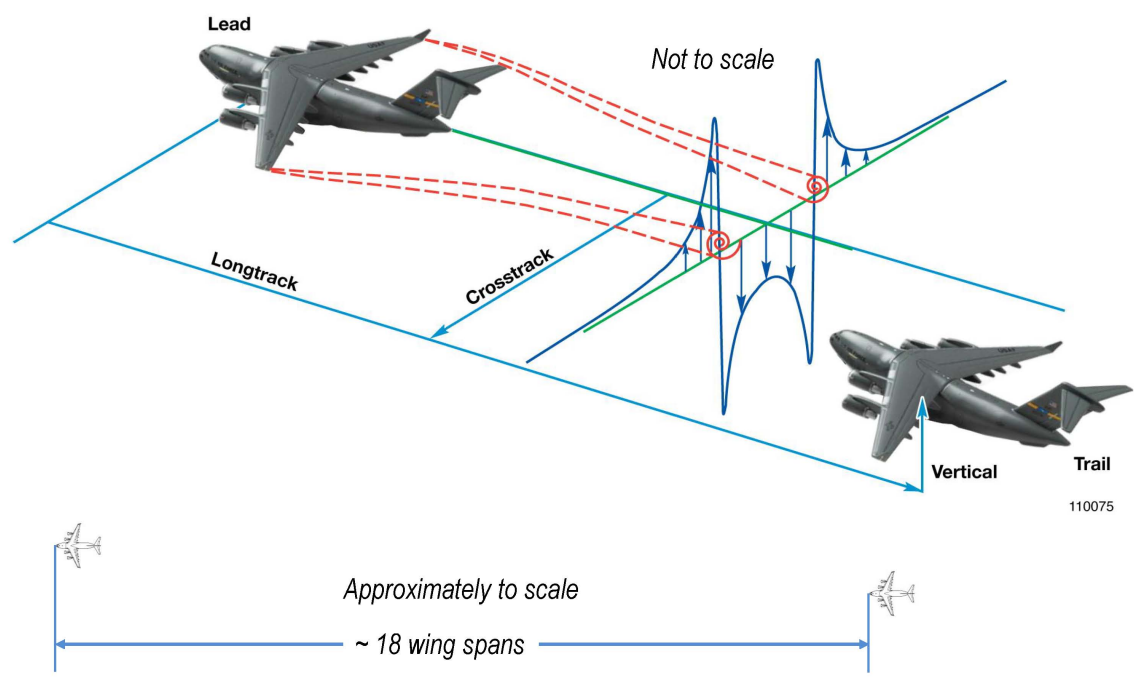

Figure 5. The NASA extended formation flight experiment using C-17 transports 9

In the subsequent designs studies we assume that the streamwise separation is 20 spans. The $y$ and $z$ offset between the wing tip and the wake are initially assumed to be 0 . Implicit is an assumption that we can accurately track the wake development in flight. This represents a significant assumption. Practical, precise, and lightweight airborne LIDAR and laser-acoustic wake tracking systems remain areas of active research. ${ }^{[2]}$ A multitude of sensor, control, and safety issues will therefore have to be addressed before extended formation flight becomes practical in commercial fleet service. Nonetheless, a recent NASA extended formation flight experiment using C-17 transports demonstrated an average trailing aircraft fuel savings of 4-5\%9 . The experiment relied upon the C-17 autopilot for station keeping and showed that savings are possible even without active wake tracking. The experimental setup illustrated in Figure 5 shows a longitudinal separation of 18 spans, which is comparable to the longitudinal separation in the current analysis.

Finally, we use the semi-empirical methods from the Program for Aircraft Synthesis Studies (PASS) to estimate the parasite, compressibility, up-sweep, and viscous lift-dependent drag for the up-and-away flight segments.23|24 The models are made numerically smooth to ensure convergence under gradient-based optimization. 


\section{Heuristic Formation Search}

The formation flight scheduling problem is NP-hard: for $n$ direct flights there are $n(n-1) 2$-aircraft and $n(n-1)(n-2) 3$-aircraft formations. However, a large portion of the set of all possible flights are not viable. For example, aircraft flying in the opposite directions are not good candidates for formation flight. Neither are aircraft whose flight times do not overlap. To increase the efficiency of the optimization we develop heuristics to identify good candidates for formation flight. The heuristics acts as filters on the combinatorial set of all possible formations.

First, we require the departure azimuth $\phi_{d}$ between aircraft $i$ and $j$ in the same formation to be bounded by $\Delta \phi_{i j}$, measured between great circle paths. The same angular limit is applied to $\phi_{a}$, the arrival azimuth. The azimuth constraints in Equation (4) eliminate formations composed of aircraft traveling in significantly different directions. For consistency, $\Delta \phi_{i j}$ is always the minor angle.

$$
\begin{aligned}
\left|\phi_{a i}-\phi_{a j}\right|<\Delta \phi_{i j} \\
\left|\phi_{d_{i}}-\phi_{d_{j}}\right|<\Delta \phi_{i j}
\end{aligned}
$$

Second, we require the sum of the distance between the departure and arrival airports $d_{d i j}$ and $d_{a i j}$ to be small relative to the sum of the flight distances $d_{i}$ and $d_{j}$. The formation "aspect ratio" rule in Equation (5) favors the combination of clustered departure airports, clustered arrival airports and extended flight distances.

$$
\frac{d_{d i j}+d_{a i j}}{d_{i}+d_{j}}<\kappa_{d}
$$

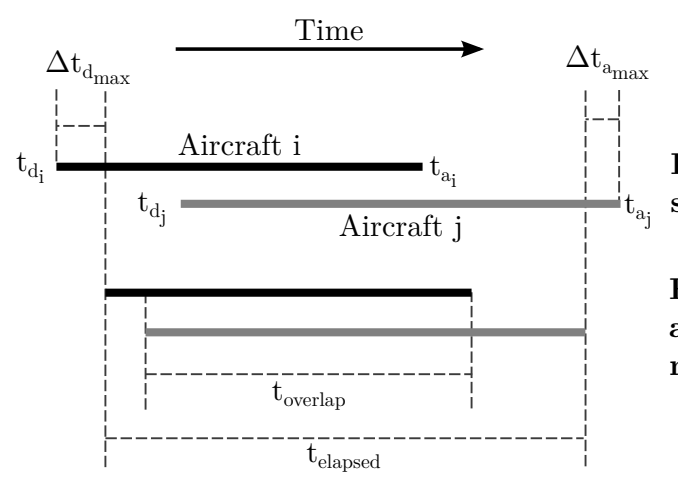

Flight timelines as originally scheduled

Flight timelines with departure and arrival flexibilities that maximize overlap

Figure 6. A timeline illustration that highlights the formation overlap parameter.

Finally, we reason that flights in acceptable formations should have finite time overlap. Two aircraft cannot fly in formation if one lands before the other can takes off. We define the overlap parameter $\kappa_{t}$ as the ratio of maximum overlapped flight time $t_{\text {overlap }}$ to the minimum elapsed time $t_{\text {elapsed }}$. A high overlap ratio is beneficial for formation flight. Figure 6 illustrates the overlap ratio in the context of flight timelines. The overlap parameter can be written as:

$$
\frac{t_{\text {overlap }}}{t_{\text {elapsed }}}=\frac{\min \left(t_{a i}, t_{a j}\right)-\max \left(t_{d i}, t_{d j}\right)+\Delta t_{d \max }+\Delta t_{a \max }}{\max \left(t_{a i}, t_{a j}\right)-\min \left(t_{d i}, t_{d j}\right)-\Delta t_{d \max }-\Delta t_{\text {amax }}}>\kappa_{t}
$$

The overlap parameter in Equation (6) is affected by the scheduling flexibility. Non-zero $\Delta t_{d \max }$ or $\Delta t_{a \max }$ increase the overlap parameter, which can be greater than unity for highly flexible schedules. The time flexibility on both the departure and arrival ends are used to maximize the overlap.

The overlap parameter, like the other heuristics, needs to be tuned. Too high an overlap requirement can eliminate good formations. We conduct sensitivity studies on the individual heuristics using reference schedules to ensure that they do not remove promising formations for optimization. In each case the heuristics greatly reduce the number of candidate formations without greatly changing the optimized schedules. This conservative property does not, however, hold in general; validations are required for different schedules.

\section{Mission Optimization}

The candidate formations are individually optimized for minimum cost or fuel burn. The large number of candidate formations and mission design variables makes the mission optimization the most expensive 
part of the routing problem. Here we use gradient-based optimization (via fmincon in MATLAB) to reduce computational cost.

Each route is parameterized using three cruise segments. We integrate for the segment range using the Breguet range equation while assuming linearly varying range factors. The range factors are computed at the beginning and end of each segment. Other segments such as takeoff, climb, descent, and approach are modeled more simply using energy arguments.

In the single-aircraft mission illustrated in Figure 7 the cruise segments model step climbs to maximize cost or fuel efficiency. The design variables include the altitude, weight, and Mach numbers at the start and end of each cruise segment. The aircraft is constrained to produce sufficient thrust to maintain steady flight at each state. It is also constrained to have enough fuel to complete each segment.

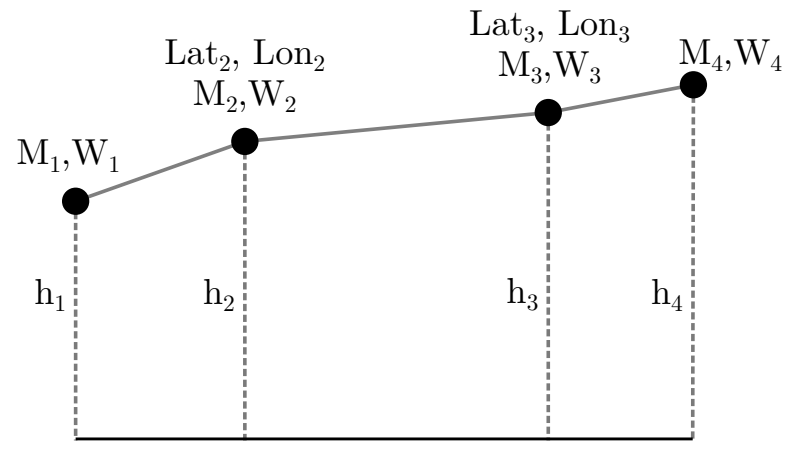

Figure 7. Solo mission parameterization.

The formation cruise mission illustrated in Figure 8 is parameterized as a combination of several solo routes with a common middle segment flown in formation. Aircraft in formation share the same Mach number and altitude at states 2 and 3 :

$$
\begin{aligned}
h_{2 i} & =h_{2 j}, \quad h_{3 i}=h_{3 j} \\
M_{2 i} & =M_{2 j}, \quad M_{3 i}=M_{3 j}
\end{aligned}
$$

A 2-aircraft formation mission is illustrated in Figure 9 Each node represents a flight state defined by longitude, latitude, and altitude. Each segment represents a great circle track. The departure and arrival coordinates are defined by the flight schedule. The flight segments are parameterized in the same way as the solo mission. The coordinates of the rendezvous and separation points are now design variables.

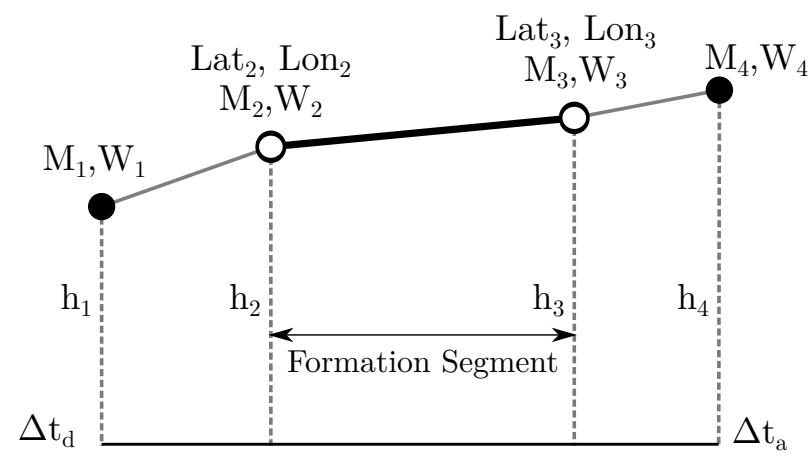

Figure 8. Formation mission parameterization. The bold segment between state 2 and 3 is flown in formation. 


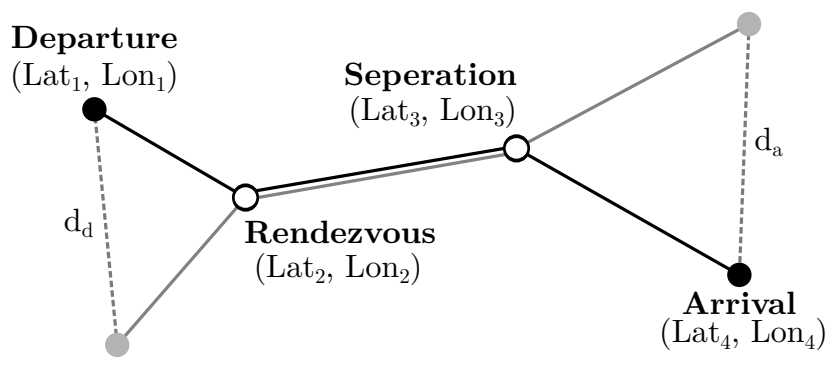

Figure 9. A schematic representation of a 2-aircraft formation mission. The line segments represent great circle paths. The segment from state 2 to 3 is flown in formation.

\section{A. Objective}

The objective of the mission optimization is to minimize either the fuel burn $\left(J_{f}\right)$ or the cost $\left(J_{c}\right)$ of a mission flow by $n_{a}$ aircraft:

$$
\begin{aligned}
& J_{f}=\min \sum_{i=1}^{n_{a}}\left(W_{1 i}-W_{4 i}+W_{f_{c i}}\right) \\
& J_{c}=\min \sum_{i=1}^{n_{a}} D O C_{i}
\end{aligned}
$$

The formation fuel burn is estimated as the sum of the changes in aircraft weight from the beginning to the end of each flight. For simplicity, the approach and landing stages are assumed to have the same specific range as the cruise segment. The takeoff and climb fuel are combined $\left(W_{f_{c}}\right)$ and estimated as a function of the aircraft state at the start of cruise:

$$
W_{f_{c}}=f\left(W_{1}, h_{1}, M_{1}\right)
$$

The direct operating cost captures the impact of block time on airline economics. Formation flight can reduce fuel burn, but it can also increase block time. There are several reasons for this: 1) in general aircraft have to divert from their shortest great circle flight path to get into formation, 2) under limited schedule flexibility aircraft may have to slow down to meet other aircraft in formation, and 3) a formation can only fly as fast as its slowest member. If fuel prices are sufficiently low then the speed penalties associated with formation flights can outweigh the cost savings from reduced fuel burn.

We estimate the aircraft direct operating cost empirically as the sum of costs that scale with the flight time, block time, and fuel burn. ${ }^{25}$ Equation $(7)$ summarizes the form of the DOC model and its sensitivity to aircraft and operational parameters.

$$
\begin{aligned}
D O C & =c_{\text {blk }} t_{\text {blk }}+c_{f t t} t_{f l t}+c_{\text {fuel }}\left(W_{1}-W_{4}\right) \\
c_{\text {blk }} & =f\left(n_{\text {cabin }}, n_{\text {cockpit }}, W_{M T O W}, k_{\text {inflate }}\right) \\
c_{f l t} & =f\left(W_{\text {airframe }}, W_{\text {engines }}, T_{0}, c_{\text {labor }}, c_{\text {oil }}, k_{\text {inflate }}\right)
\end{aligned}
$$

We assume that the costs of depreciation, insurance, per-flight maintenance, and landing fees are identical for aircraft flying in and out of formations. The cost analysis is based on an assumed fuel cost $c_{f u e l}$ of $\$ 3.30 /$ gal and a maintenance labor rate $c_{\text {labor }}$ of $\$ 40 /$ hour. This simple analysis cannot precisely predict absolute economic performance. We can, however, use it to compare the relative cost performance of formation and solo scheduling.

\section{B. Variables}

The mission optimization design variables can be divided into the solo $\left(\boldsymbol{x}_{\boldsymbol{s}}\right)$ and formation $\left(\boldsymbol{x}_{\boldsymbol{f}}\right)$ components. The former are defined for each aircraft:

$$
\boldsymbol{x}_{\boldsymbol{s}}=\left[h_{1}, h_{4}, M_{1}, M_{4}, W_{1}, W_{2}, W_{3}, W_{4}, \Delta t_{d}, \Delta t_{a}\right]
$$

The aircraft weight at each flight state is not converged using fixed-point iteration. Rather, the weights are posed as variables and their values converged as part of the overall mission optimization. The range 
constraints discussed in Section C ensure that the change in weight between successive flight states (the segment fuel burns) are sufficient to cover the segment distance.

The parameters $\Delta t_{d i}$ and $\Delta t_{a i}$ specify the changes in departure and arrival times for aircraft $i$. The time flexibility allows aircraft to better divert, slow down or speed up to rendezvous with other aircraft. The remaining variables are defined for each formation:

$$
\boldsymbol{x}_{\boldsymbol{f}}=\left[h_{2}, h_{3}, M_{2}, M_{3}, \text { lat }_{2}, \text { lat }_{3}, \text { lon }_{2}, \text { lon }_{3}\right]
$$

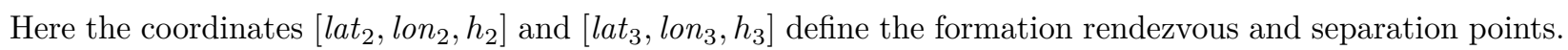
Aircraft in formation share the state variables at point 2 and 3.

\section{Constraints}

The mission optimization is subject to a combination of range, drag, and time constraints defined at the different flight states. We use a simplified model to obtain the aircraft performance and constraint violations. Many of the method are derived from the Program for Aircraft Synthesis Studies (PASS) ${ }^{23}$ The physics-based induced drag factors discussed in Section II accounts for the effects of formation flight.

First, the aircraft must meet a thrust margin constraint at each flight state. It mush also have enough range to fly through each of its cruise segments $k$ :

$$
\begin{aligned}
\left(\frac{D}{T}\right)_{\text {solo }} & <0.88 \\
d_{k} & <r_{k}
\end{aligned}
$$

In the case of formation flight the range constraint includes the induced drag benefits from formation flight. The thrust margin on the other hand is always based on solo operation and ignores the effects of formation flight. This ensures that aircraft in formation retain independent operational climb capabilities.

The available engine thrust and TSFC are computed using a rubberized PW2037 turbofan deck. TSFC and thrust-to-weight correction factors are used to adjust the deck to emulate the performance of more modern engines.

To obtain range performance we first trim the aircraft and compute the wing and horizontal tail $C_{L}$. Next, the inviscid component of the induced drag is computed based on elliptical wing loads and a semi-empirical estimate of fuselage and horizontal tail interference drag. The aircraft inviscid $C_{D i}$ is multiplied by the appropriate formation induced drag factor discussed in Section III The parasite drag is computed using equivalent plate area methods based on component form factors. ${ }^{23}$ The component $C_{f}$ are corrected for compressibility effects. Finally, the aircraft compressibility drag is estimated using the semi-empirical method of McGeer and Shevell. ${ }^{24}$ There is no explicit accounting of any additional compressibility drag that may come from flying in formation.

In addition to the formation range constraints, we also require each aircraft in formation to carry enough fuel to complete their mission without any formation drag benefits:

$$
d_{k}<r_{k s o l o}
$$

In a conservative implementation of formation flight, the contingency range constraint supersedes the formation segment range constraint in Equation (8). This additional fuel margin anticipates the worst-case scenario in which an aircraft commits to a longer formation mission, but fails to achieve any fuel savings. In this event, the aircraft in question should still be able to reach its destination. We pose the solo contingency mission as a constraint. Aircraft carry the extra fuel needed to complete the mission solo, but do not burn this reserve in formation operations. This conservative realization of formation flight reduces the fuel burned, but not the fuel carried. In fact, since formation missions often involve diversions from the direct great circle route, an aircraft may have to carry more fuel for the formation mission than the more direct, solo mission. The weight penalties of the additional reserve is non-trivial. In the 31-aircraft schedule optimization study in Section VI the reserve requirement wipes out $25 \%$ of the cost and $20 \%$ of the fuel savings from formation flight. If formation flight proves reliable, then contingency airports could be identified prior to flight, and diversions to these airports could be used if formation flight is not possible. This would alleviate this constraint, reduce fuel carried, and even further reduce fuel burned. 
Each aircraft's departure and arrival times are constrained to lie within $\Delta t_{d}$ and $\Delta t_{a}$ of the scheduled times:

$$
\begin{aligned}
& t_{a}-\Delta t_{a}<t_{4}<t_{a}+\Delta t_{a} \\
& t_{d}-\Delta t_{d}<t_{1}-t_{c}<t_{d}+\Delta t_{d}
\end{aligned}
$$

Where $t_{c}$ is an estimated climb time. The total flight time is further constrained to lie within some $\Delta t_{f}$ of the scheduled flight time:

$$
t_{a}-t_{d}-\Delta t_{f}<t_{4}-\left(t_{1}-t_{c}\right)<t_{a}-t_{d}+\Delta t_{f}
$$

Here $\Delta t_{a}, \Delta t_{d}$, and $\Delta t_{f}$ capture the effect of schedule flexibility on the efficiency of formation flight. Greater flexibility increases the number of feasible formations, and reduces the need for aircraft to fly at non-optimal speeds to reach formation. The formation missions are further subject to equality constraints on rendezvous and separation time:

$$
\begin{aligned}
& t_{2 i}=t_{2 j} \\
& t_{3 i}=t_{3 j}
\end{aligned}
$$

The outcome of the mission optimization is a set of individually optimal formation and solo missions. This set does not, in general, form a consistent schedule - one aircraft can appear in multiple missions. Integer programming is used to find the consistent and optimal schedule of candidate formations.

\section{Schedule Optimization}

The objective of the schedule optimization is to find the best combination of formation and solo missions. For this we use the MATLAB binary integer programming tool bintprog. The schedule optimization takes only seconds on a modern computer. The optimization problem can be posed as follows:

$$
\begin{array}{ll}
\text { minimize } & J_{\text {schedule }}=\sum_{k=1}^{3} \boldsymbol{J}_{\text {mission } k}{ }_{k}^{T} \boldsymbol{x}_{\text {schedulek }} \\
\text { w.r.t. } & {\left[\boldsymbol{x}_{\text {schedule } 1}, \boldsymbol{x}_{\text {schedule } 2}, \boldsymbol{x}_{\text {schedule } 3}\right]} \\
\text { s.t. } & \sum_{k=1}^{3} \boldsymbol{A}_{\text {schedule } k} \boldsymbol{x}_{\text {schedule }}=\boldsymbol{e} \\
\boldsymbol{J}_{\text {missionk }}= \begin{cases}\left\{J_{c}\right\}_{k} & \text { minimum cost } \\
\left\{J_{f}\right\}_{k} & \text { minimum fuel }\end{cases}
\end{array}
$$

The schedule optimization objective $J_{\text {schedule }}$ is the sum of the $D O C$ or fuel burn of all of the aircraft in the schedule. We compute $J_{\text {schedule }}$ using the vector of optimized candidate mission objectives $\boldsymbol{J}_{\text {missionk }}$. The mission objectives are organized by formation size: the index $k$ differentiates between solo $(k=1)$, two aircraft $(k=2)$ or three aircraft formations $(k=3)$. The binary decision vectors $\boldsymbol{x}_{\text {schedule }}$, also organized by formation size, control which solo, 2-aircraft or 3-aircraft mission is flown. $\boldsymbol{x}_{\text {schedule }}$ is of size $n_{m k}$ by 1 , where $n_{m k}$ is the number of optimized candidate missions for formation size $k$. An element of $\boldsymbol{x}_{\text {schedulek }}$ is 1 if the mission is flown and 0 otherwise.

The schedule optimization is subject to the constraint that every route in the schedule is flown exactly once. This constraint is posed using the $n_{a}$ by $n_{m k}$ mission mapping matrices $\boldsymbol{A}_{\text {schedulek }}$. Recall that $n_{a}$ denotes the number of aircraft in the schedule. An element of $\boldsymbol{A}_{\text {schedule }}$ is 1 if flight $i$ is contained in mission

$j$ and 0 otherwise. The solo mission mapping matrix $\boldsymbol{A}_{\text {schedule } 1}$, for example, is an $n_{a}$ by $n_{a}$ identity matrix. The constraint function counts therefore how many times each flight is flown in the optimized schedule. For a self-consistent schedule the constraint function must produce a vector of 1's, which we denote as $\boldsymbol{e}$.

\section{South African Airlines Study}

We optimize two representative airline schedules to quantify the system-level benefits of extended formation flight. The first study is based on the 31-flight South African Airway (SAA) long-haul route network from October 2009, which is shown in Figure 10. A fleet of Airbus A330-200, A340-200/300/600, and Boeing 747-400 aircraft fly the SAA schedule. 


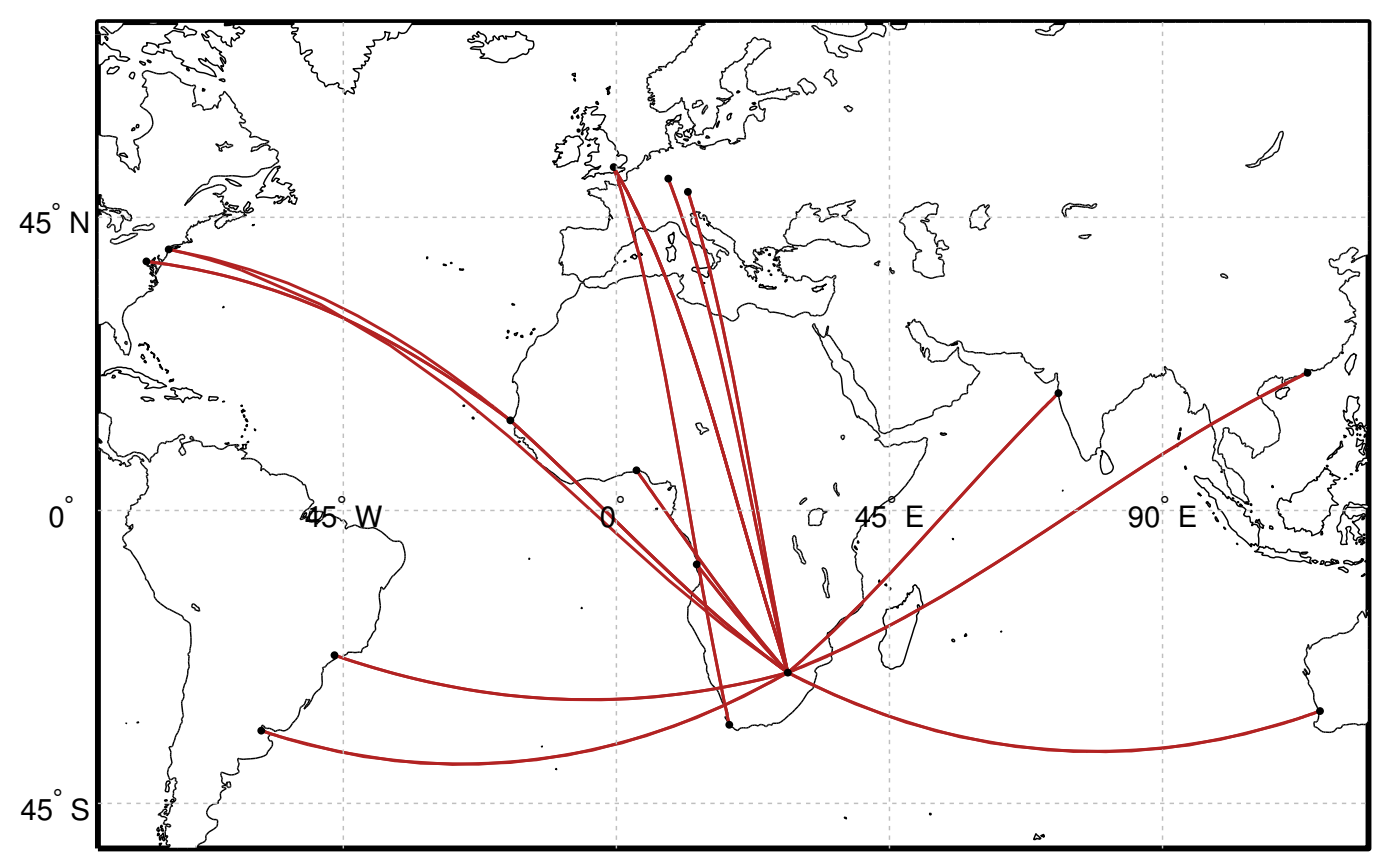

Figure 10. The baseline South African Airway (SAA) route network.

Table 1 summarizes the heuristics used to find the candidate formations. We use relatively unrestrictive heuristics for this small problem. They allow for a generous one-hour flexibility in arrival and departure time. The departure and arrival azimuth differences can be up to $120^{\circ}$. The study also assumes that the wing tips of the trailing aircraft are aligned with the center of the wake vortex $(\Delta y=0)$. The overlap parameter at 0.7 is unrestrictive given the flexibility in arrival and departure time. A sensitivity study shows that changing the overlap ratio in the range of 0.5 to 0.9 has essentially no impact on formation performance. This result is however, particular to the SAA formation.

$$
\begin{array}{lll}
\kappa_{d}=0.4 & \Delta x=20 b & \Delta \phi_{i j}=120^{\circ} \\
\kappa_{t}=0.7 & \Delta y=0 & \Delta t_{a i}=1 \text { hour } \\
& \Delta z=0 & \Delta t_{d i}=1 \text { hour }
\end{array}
$$

Table 1. The SAA heuristic formation search filter.

Figures 11 and 12 show the minimum cost and minimum fuel SAA schedules. Their structures are similar.

Table 2 shows that the same number of 2 and 3-aircraft formations are flown for the minimum cost and minimum fuel objectives. Aircraft tend to spend a greater percentage of flight time in formation for the minimum-fuel study: $57 \%$ versus $54 \%$. This result is intuitive. If fuel burn were the only objective then the savings from formation flight can justify significant diversions from the baseline great circle route. However, if the goal is to minimize cost then the fuel savings have to be weighed against the increased block time needed to get in and out of formation.

A formation schedule that minimizes fuel burn can save $5.8 \%$ in fuel and $1.3 \%$ in cost compared to the minimum cost solo schedule. Alternatively, a minimum DOC formation schedule can save $4.8 \%$ in fuel and $2.0 \%$ in cost. These savings compare favorably against vehicle technologies like transonic natural laminar flow wings 26$] 28$

\section{A. SAA Compressibility Effect Study}

The drag reductions from formation flight are realized by flying trailing aircraft in the upwash of leading aircraft wake(s). At transonic speeds the increased local angles of attack from the wake upwash can cause stronger-than-usual shocks on trailing aircraft. Moreover, in many formations the trailing aircraft need to trim in roll using asymmetric wing control surface deflections. Any positive deflection at high speed would 


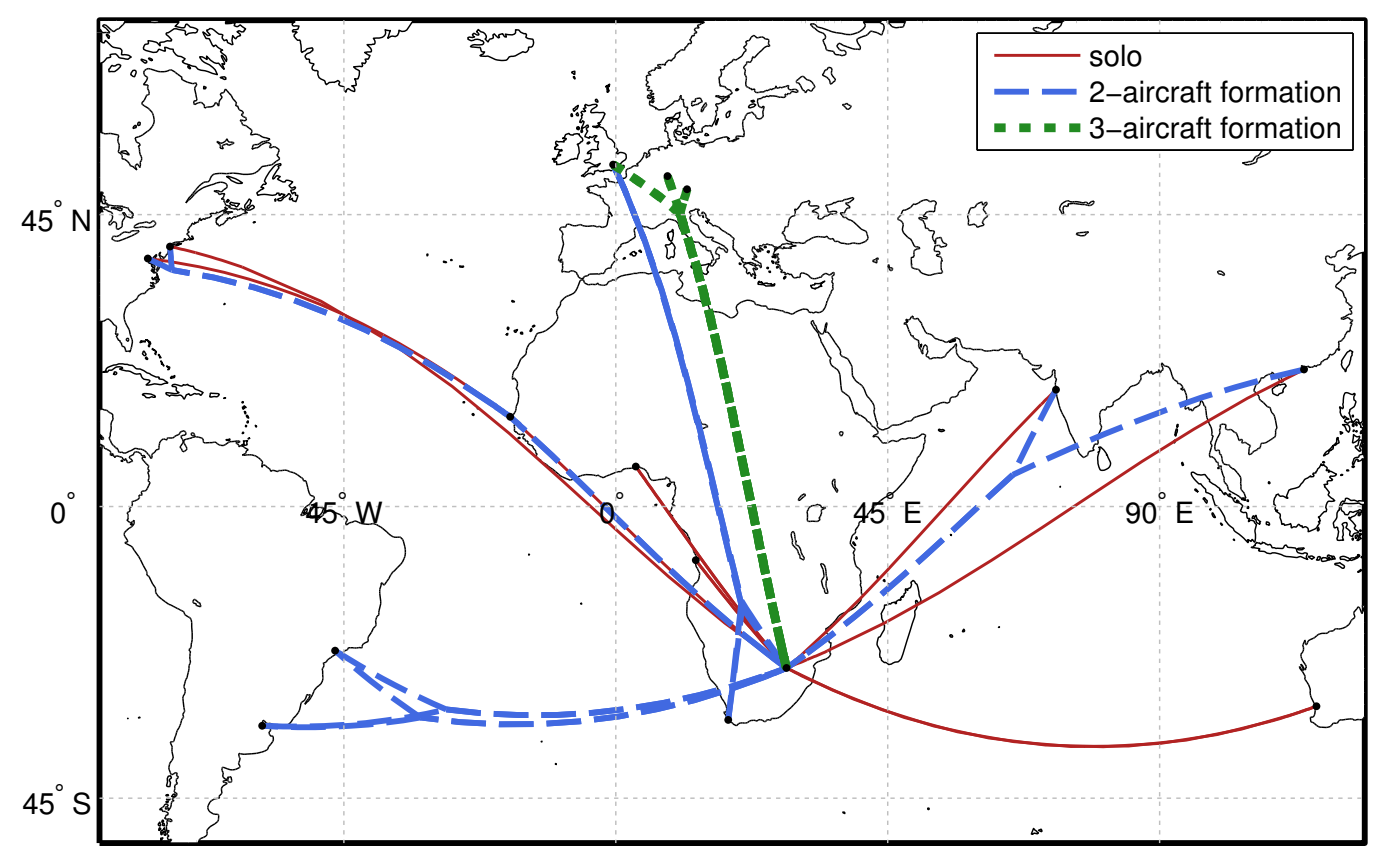

Figure 11. The SAA network optimized for minimum $D O C$ formation flight.

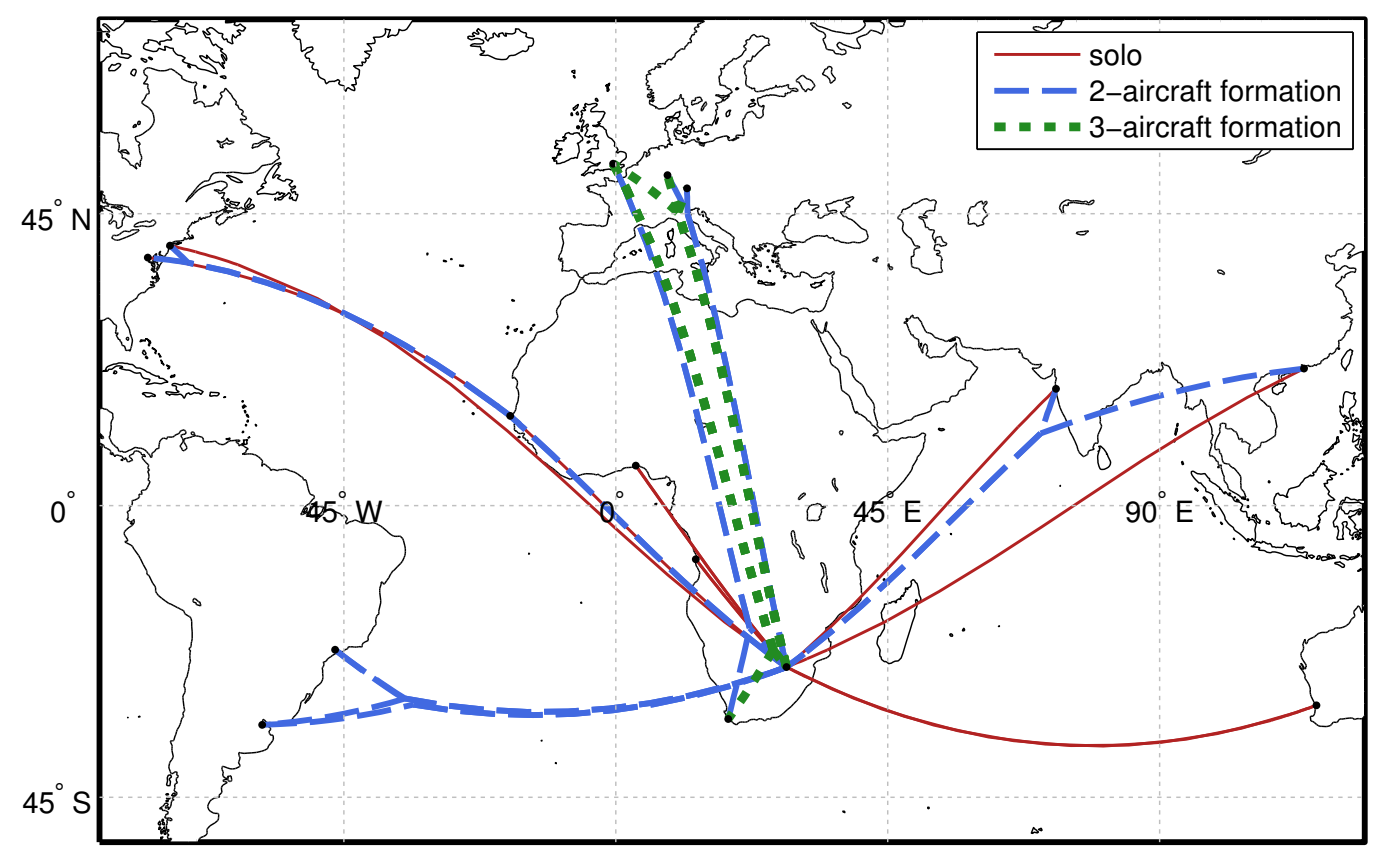

Figure 12. The SAA network optimized for minimum fuel formation flight. 


\begin{tabular}{lrr}
\hline & $\min (\boldsymbol{D O C})$ & $\min \left(\boldsymbol{W}_{\boldsymbol{f}}\right)$ \\
\hline Solo missions & 11 & 11 \\
2-Aircraft formations & 7 & 7 \\
3-Aircraft formations & 2 & 2 \\
Distance in formation & $54.1 \%$ & $56.8 \%$ \\
\hline$\Delta$ Fuel & $-4.8 \%$ & $-5.8 \%$ \\
$\Delta$ DOC & $-2.0 \%$ & $-1.3 \%$ \\
$\Delta$ Time & $2.7 \%$ & $6.9 \%$ \\
$\Delta$ Distance & $0.7 \%$ & $0.8 \%$ \\
\hline
\end{tabular}

Table 2. SAA formation minimum cost and fuel optimization results. The performance $\Delta$ are relative to the minimum cost solo schedule.

further increase shock strength. Transonic aircraft are typically designed to cruise near drag divergence to maximize cost performance. Tangible increases in the wing shock strength can lead to shock-induced flow separation.

Trailing aircraft can slow down or fly further away from the wake to cope with these additional compressibility effects. ${ }^{[21}$ However, slowing down can increase block time and hurt cost performance. Flying further from the wake can degrade formation flight savings. Compressibility can therefore limit formation flight fuel and cost performance.

We examine the performance impacts of three combinations of lateral wake offsets and cruise speed reductions designed to mitigate compressibility effects. The three mitigation strategies are summarized in Table 3. Here $\Delta M$ is defined in terms of the maximum allowable formation cruise Mach number, which is in turn dictated by the slowest aircraft in the formation. The $\Delta y$ separation is defined in terms of the span of the leading aircraft. The speed reduction and lateral offset combinations are selected based on Euler analyses of wake propagation and formation flight conducted by Ning. ${ }^{13}$ The three strategies are estimated to have roughly the same compressible drag penalty as the same aircraft flying alone near its drag-divergence Mach number.

\begin{tabular}{lrr}
\hline & $\boldsymbol{\Delta} \boldsymbol{M}$ & $\boldsymbol{\Delta} \boldsymbol{y}$ \\
\hline Slow & $-2.5 \%$ & 0 \\
y-offset & 0 & $0.10 b$ \\
Combination & $-1.0 \%$ & $0.05 b$ \\
\hline
\end{tabular}

Table 3. Three formation flight strategies to cope with the compressibility effects of leading aircraft upwash.

The optimized routes of the three compressibility mitigation strategies are shown in Fig. 13 to 15 . The route structures are markedly different. Table 4 shows that a $2.5 \%$ reduction in the maximum cruise Mach number does not alter the number of formations relative to the baseline minimum cost formation schedule. A $10 \%$ y-offset from the wake on the other hand significantly reduces the number of formations and drag savings.

The results also show significant cost and fuel burn penalties if we use only y-offset to manage compressibility effects. On the other hand, the cost and fuel penalty associated with slowing down by $2.5 \%$ is negligible. The fuel consumption is virtually unchanged from the minimum cost formation network. This result is intuitive: slowing down has a positive effect on fuel burn while increasing lateral offset always reduces the drag savings. Moreover, the network optimization and schedule flexibility present additional degrees of freedom to make up for the Mach number reduction at the system level. The ability to change altitudes also gives the mission optimization more flexibility to cope with Mach number limits. 


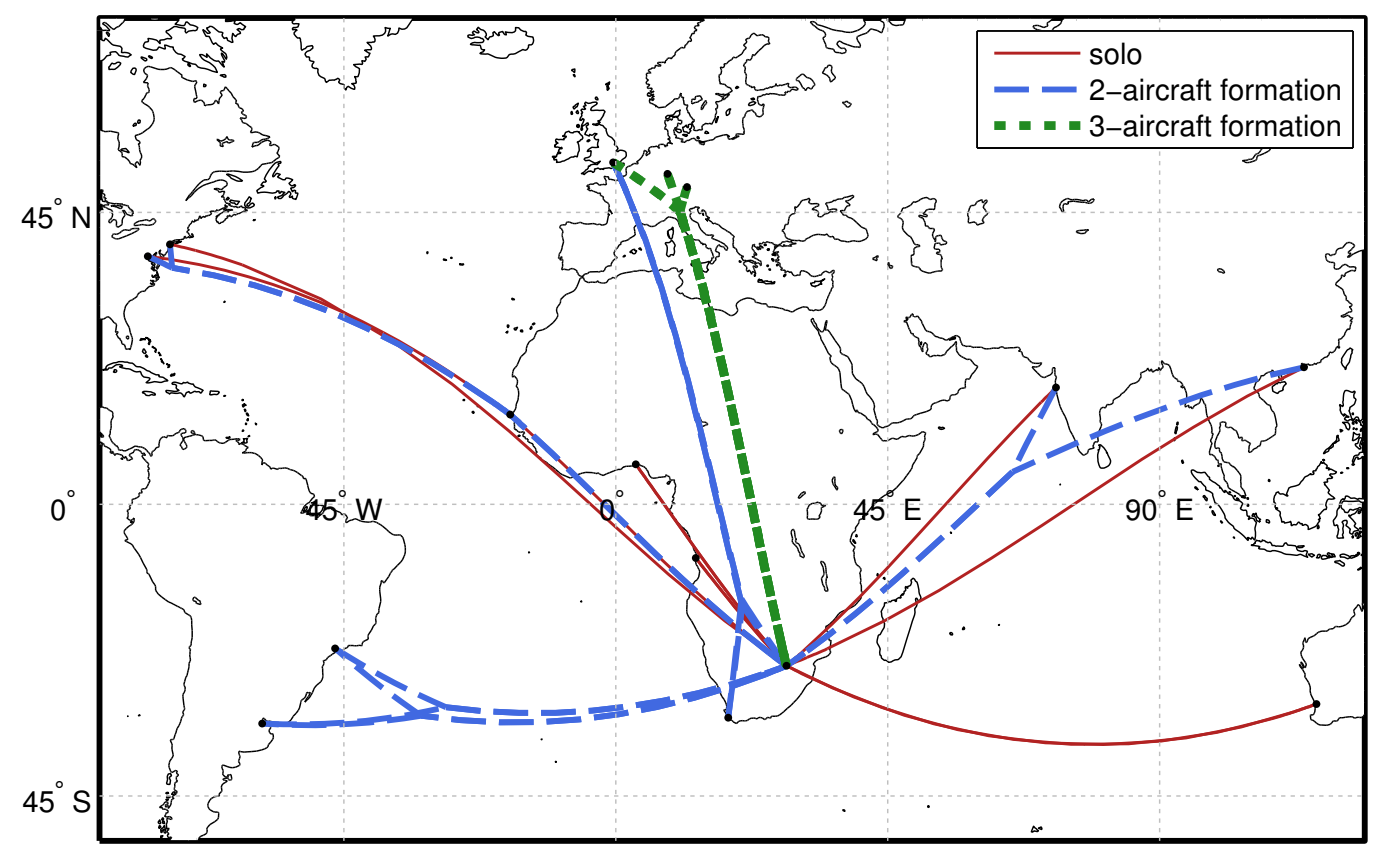

Figure 13. The minimum cost SAA formation network with a $2.5 \%$ reduction in the maximum allowable formation cruise Mach number.

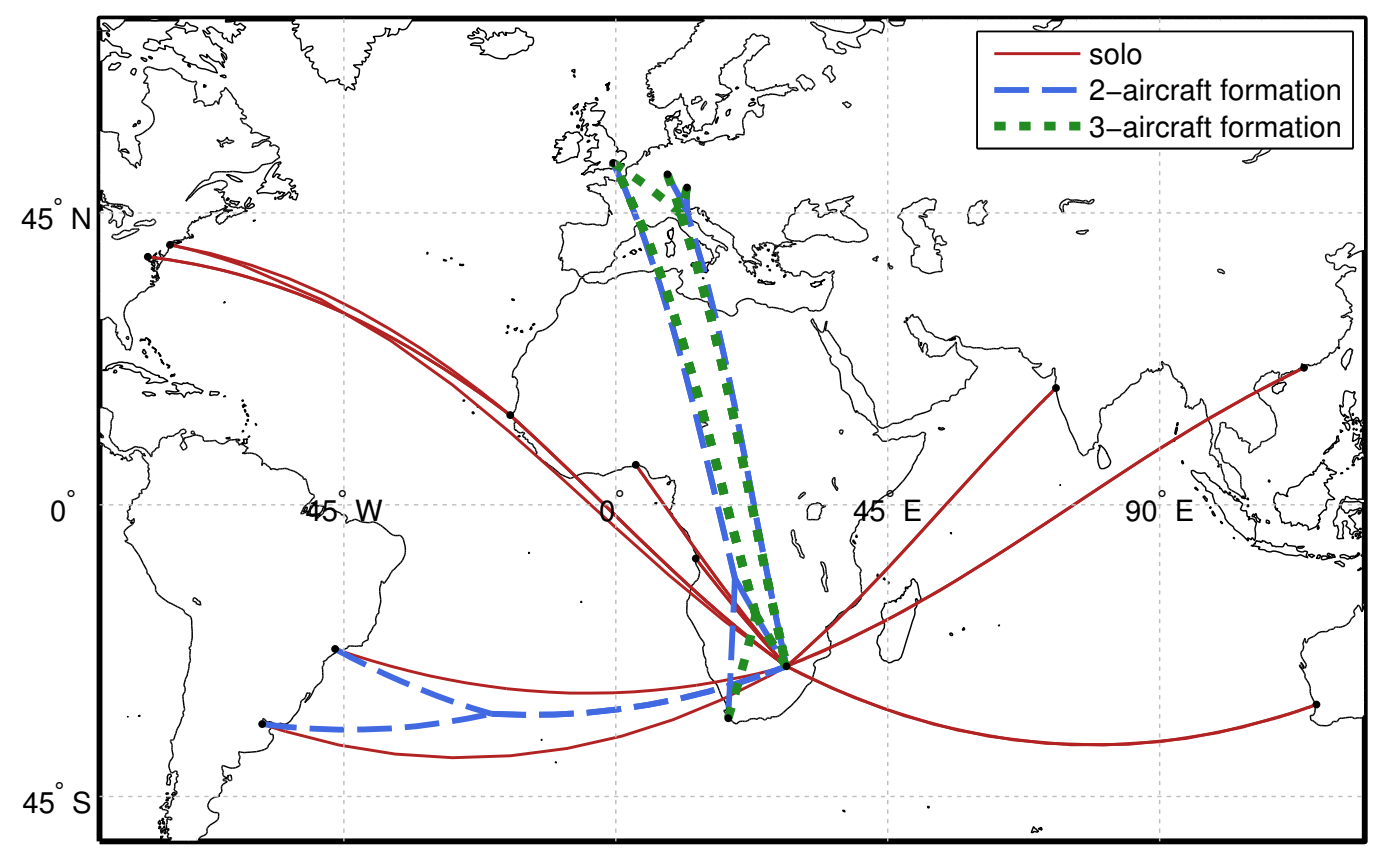

Figure 14. The minimum cost SAA formation network with a tip separation of $0.10 b$. 


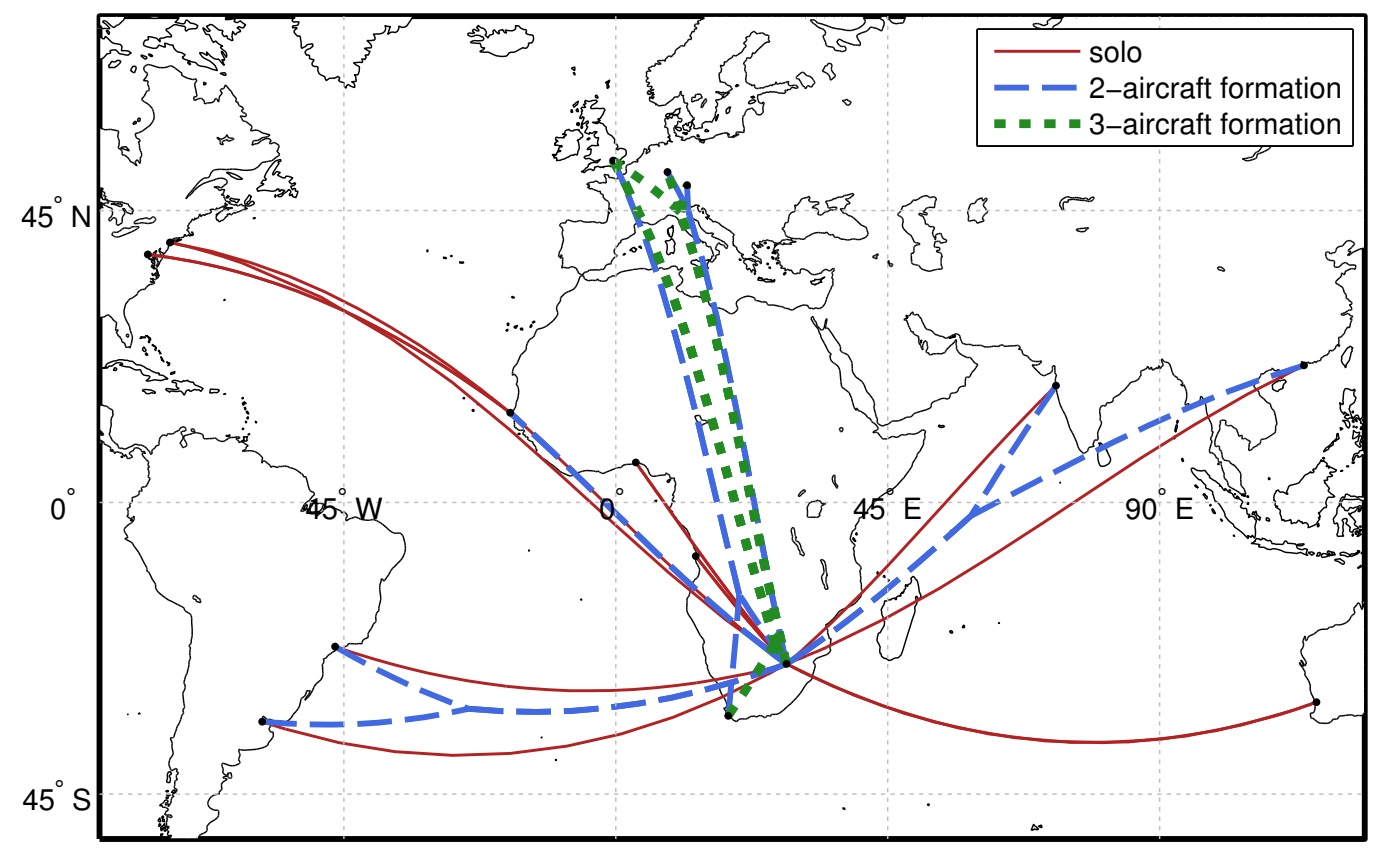

Figure 15. The minimum cost SAA formation network with a $1 \%$ reduction in the maximum allowable formation cruise Mach number and a tip separation of $0.05 \mathrm{~b}$.

\begin{tabular}{lrrr}
\hline & Slow & y-offset & Combination \\
\hline$\Delta M$ & $-2.5 \%$ & 0 & $-1.0 \%$ \\
$\Delta y$ & 0 & $0.10 b$ & $0.05 b$ \\
\hline Solo missions & 11 & 19 & 15 \\
2-Aircraft Formations & 7 & 3 & 5 \\
3-Aircraft Formations & 2 & 2 & 2 \\
Distance in Formation & $54.2 \%$ & $35.2 \%$ & $44.6 \%$ \\
\hline$\Delta$ Fuel & $-4.8 \%$ & $-1.9 \%$ & $-3.1 \%$ \\
$\Delta$ Cost & $-2.0 \%$ & $-0.9 \%$ & $-1.3 \%$ \\
$\Delta$ Time & $3.0 \%$ & $1.1 \%$ & $1.8 \%$ \\
$\Delta$ Distance & $0.7 \%$ & $0.3 \%$ & $0.5 \%$ \\
\hline
\end{tabular}

Table 4. SAA compressibility mitigation study results. The $\Delta$ in the performance metrics are defined relative to the minimum cost solo schedule. 


\section{Star Alliance Design Study}

Single-airline formation flight is likely easier to manage than a collaborative multi-airline implementation. If multiple airlines were involved then they would need to agree on a system to distribute the cost and benefits of formation flight among leading and trailing aircraft, which can now come from different airlines. There are good reasons; however, to consider formation flight for multiple airlines. Foremost of these is that more aircraft flying similar routes will lead to more and better formations.

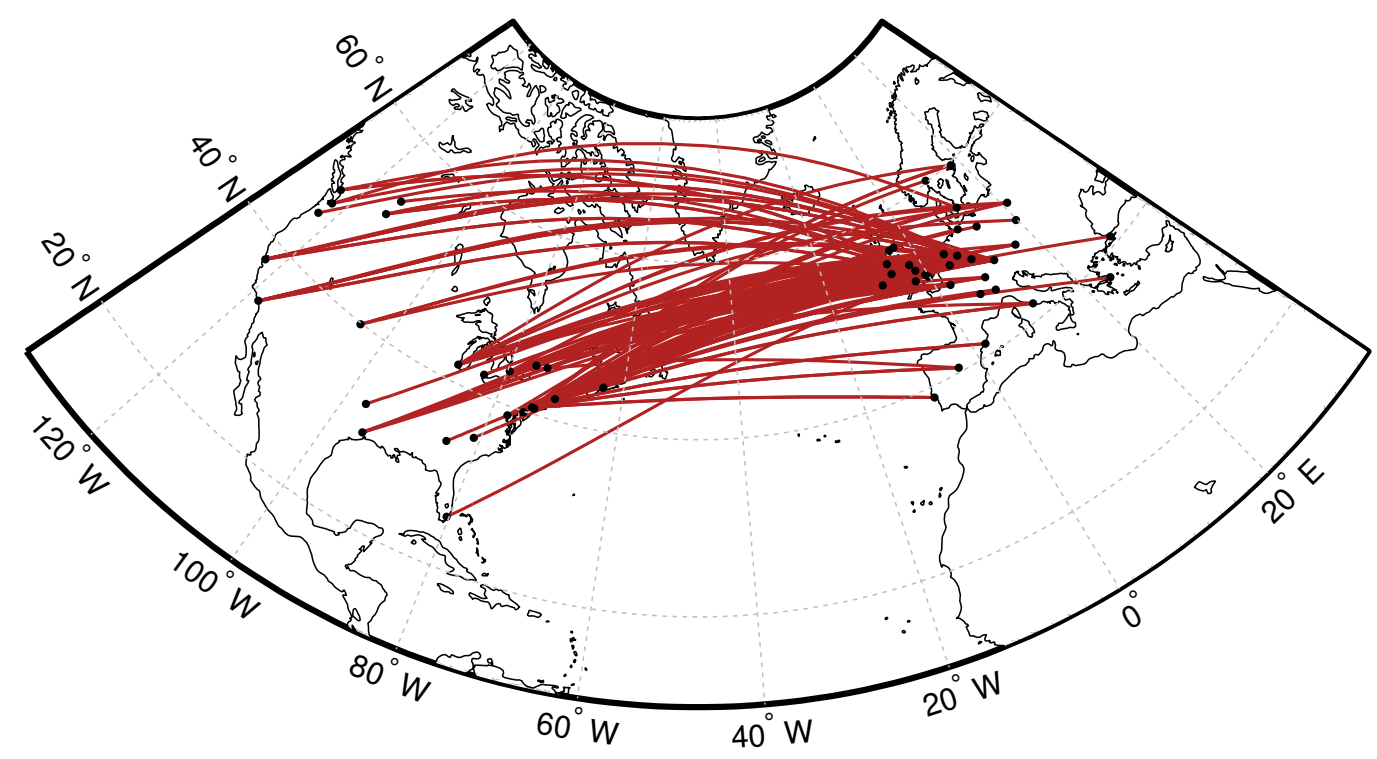

Figure 16. The Star Alliance transatlantic route network used in the design study.

Airline alliances and code/profit sharing arrangements can provide the institutional framework for managing large-scale, multi-airline formation flight. We apply the formation flight optimization framework developed in the previous sections to a 150-flight, two-hour snapshot of an eastbound Star Alliance transatlantic flight schedule. The Star Alliance route network shown in Figure 16 is served by 12 types of Airbus and Boeing aircraft.

For a 150-aircraft schedule there are 16,770 possible two-aircraft and 2,146,560 possible three-aircraft formations. To make the problem more tractable we use the restrictive heuristics listed in Table 5 to identify candidate formations.

\begin{tabular}{lll}
\hline$\kappa_{d}=0.15$ & $\Delta x=20 b$ & $\Delta \phi_{i j}=30^{\circ}$ \\
$\kappa_{t}=0.9$ & $\Delta y=0$ & $\Delta t_{a i}=6 \mathrm{~min}$ \\
& $\Delta z=0$ & $\Delta t_{d i}=6 \mathrm{~min}$ \\
\hline
\end{tabular}

Table 5. The Star Alliance heuristic filter and formation design parameters.

Significantly, we decrease the departure and arrival flexibility from the 1 hour in the SAA study to just 6 minutes. We increase the required flight overlap requirement $k_{t}$ to $90 \%$ of the total scheduled flight time. Finally, we decrease the formation aspect ratio parameter $\kappa_{d}$ discussed in Section III from 0.3 to 0.15. The large numbers of potentially good formations justifies the restrictive filters. The heuristic filter removed $97.4 \%$ and $99.7 \%$ of all possible two and three-aircraft formations. This still leaves some 2,500 formation missions to be optimized, which can take up to $200 \mathrm{CPU}$ hours on a $2.1 \mathrm{GHz}$ AMD Opteron processor. The scale of the Star Alliance problems makes it difficult to verify that the heuristic filters do not remove good formations. The resulting formation schedules are likely to be sub-optimal and therefore, conservative in their projection of savings. It should be noted that since each mission optimization is independent, the problem is naively parallel. A parallel implementation of the formation mission optimization would make the solution scalable to even larger networks.

Figures 17 and 18 show the Star Alliance route network optimized for minimum fuel burn and cost, respectively. The results include a large number of three-aircraft formations, particularly for the minimum 


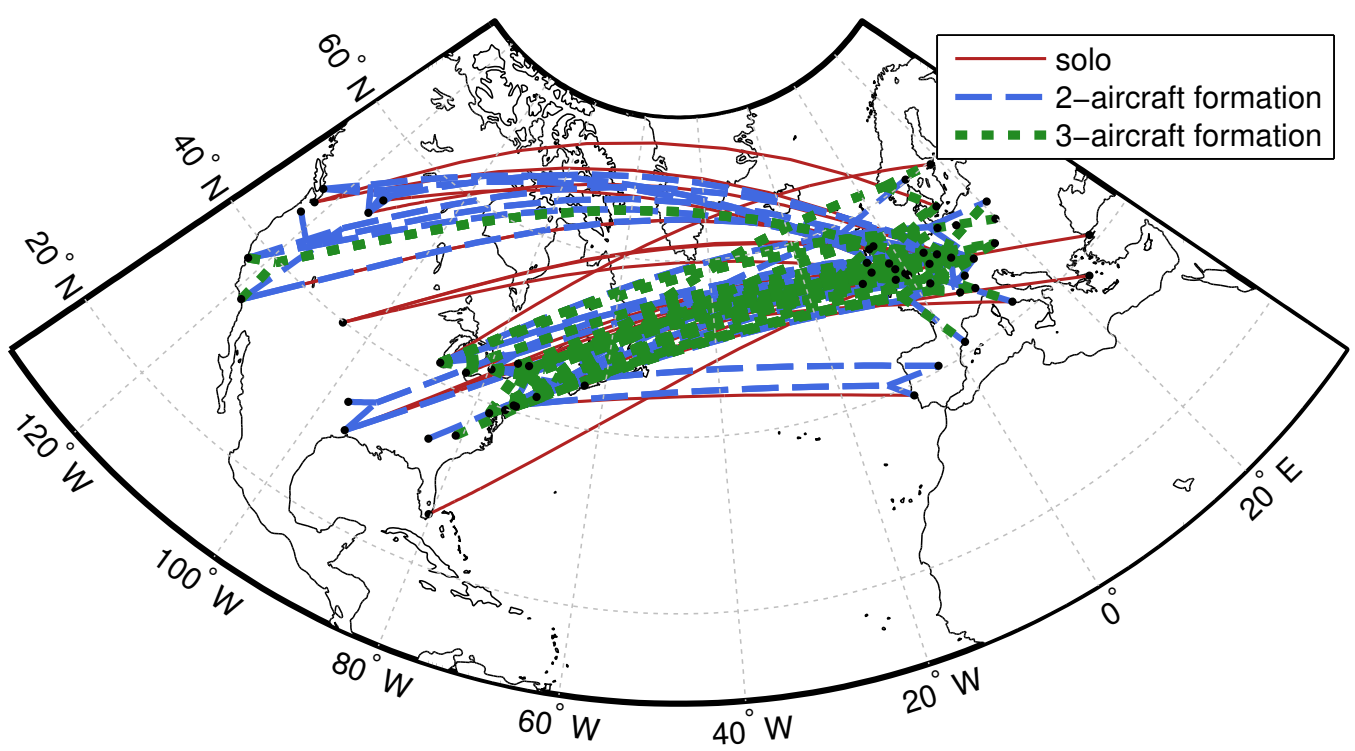

Figure 17. Star Alliance network optimized for minimum fuel formation flight.

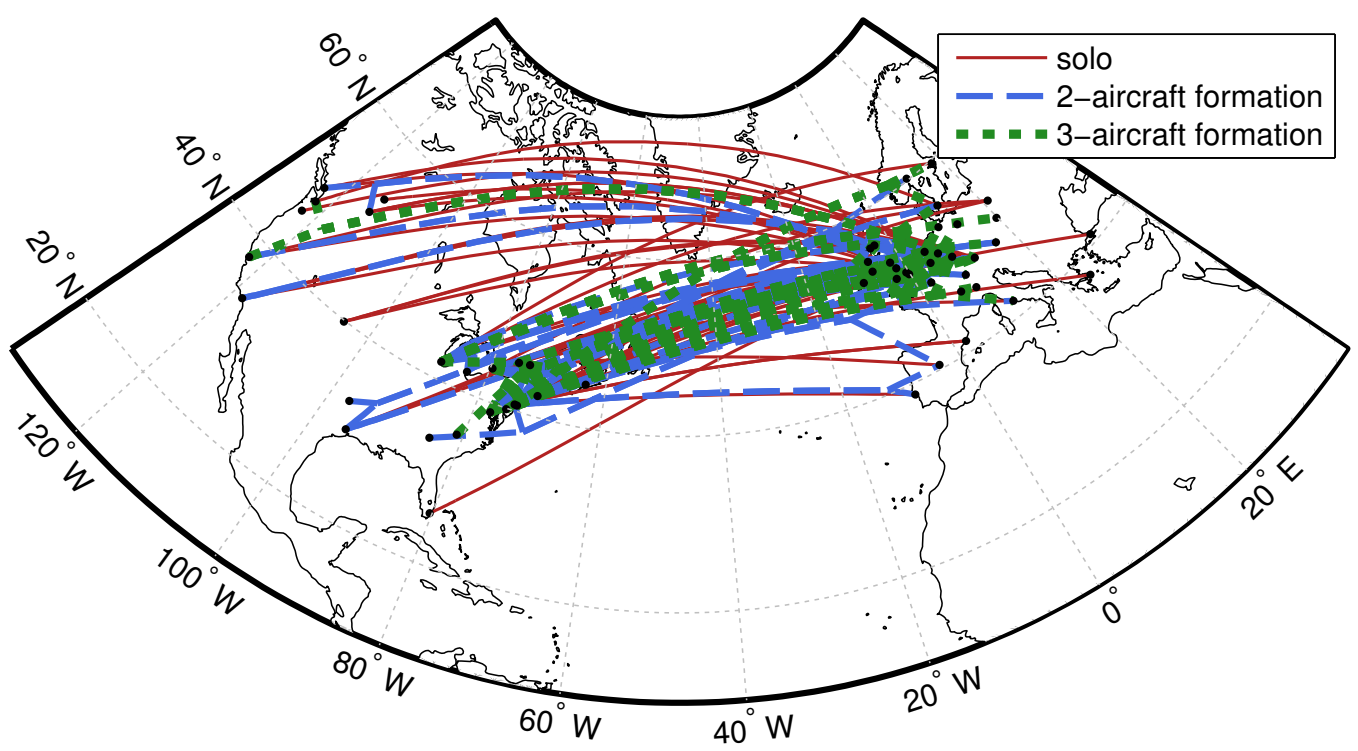

Figure 18. Star Alliance network optimized for minimum DOC formation flight. 
fuel case.

\begin{tabular}{lrr}
\hline & $\boldsymbol{m i n}(\boldsymbol{D O} \boldsymbol{C})$ & $\min \left(\boldsymbol{W}_{\boldsymbol{f}}\right)$ \\
\hline Solo missions & 37 & 23 \\
2-Aircraft Formations & 22 & 26 \\
3-Aircraft Formations & 23 & 25 \\
Distance in Formation & $61.1 \%$ & $67.5 \%$ \\
\hline$\Delta$ Fuel & $-6.9 \%$ & $-7.7 \%$ \\
$\Delta$ Cost & $-2.6 \%$ & $-2.2 \%$ \\
$\Delta$ Time & $4.9 \%$ & $7.4 \%$ \\
$\Delta$ Distance & $0.8 \%$ & $0.9 \%$ \\
\hline
\end{tabular}

Table 6. Star Alliance formation optimization results. The performance $\Delta$ are defined relative to the minimum cost solo schedule.

The results in Table 6 show that the minimum fuel formation network achieves a significant $7.7 \%$ reduction in fuel burn and a $2.2 \%$ reduction in $D O C$, compared to the minimum cost solo network. The minimum $D O C$ network reduces fuel burn and cost by $6.9 \%$ and $2.6 \%$, respectively against the minimum cost solo network. The savings are more significant than the smaller SAA network discussed in Section VI Moreover, these tangible savings are achieved with a highly restrictive departure and arrival flexibility of only 6 minutes. A large, spatially and temporally concentrated multi-airline schedule can stand to benefit greatly from extended formation flight.

\section{A. Star Alliance Delay Sensitivity Study}

The deterministic analysis presented thus far does not consider the myriad of operational disruptions that airlines face on a daily basis. Flight delays, for example, can complicate formation mission planning and reduce the potential for savings. The effect of flight and airport delays on a multi-stage schedule is complex and highly coupled. An upstream event can produce cascading downstream effects. By requiring aircraft to fly together, formation flight would increase the degrees of coupling in an already highly interactive problem. It is essential therefore to qualify the value of formation flight under scheduling uncertainty.

The objective of this paper is to ground the study of formation flight scheduling in physics-based aircraft performance and aerodynamics. The detailed stochastic analysis of delay and multi-stage operations, while undoubtedly important, is beyond the scope of this effort. However, we can still give a first-cut estimate of the impact of delays on formation flight performance by manipulating the candidate formations in the schedule optimization.

One can conservatively model a delayed aircraft as one that is unable to participate in any formation flight. The delayed aircraft flies its baseline solo mission. All formations that include the delayed aircraft are excluded from the network optimization.

Starting with the optimized candidate formations for the Star Alliance network we randomly delay a subset of the 150 flights, remove the formations that contain the delayed aircraft, and optimize the schedule. We repeat this process 4,000 times to extract statistics. This process is then repeated for different delay levels. The mean and standard deviations of the formation fuel and cost savings at different levels of random delay are plotted in Figure 19

For the Star Alliance network the formation fuel and cost savings decrease linearly with increased delays. As a reference, from 2004 to 2009 about $77 \%$ of U.S. airline flights arrived on time (defined as arriving within 15 minutes of their scheduled time) $\stackrel{[29}{\cdot}$ In our simplistic model this level of delay would result in roughly a $25-30 \%$ degradation in formation flight savings.

With less restrictive heuristics and a larger pool of candidate formations, the impact of delay should become less severe. Moreover, simply removing a delayed aircraft from formation flight is conservative. A more dynamic scheduling system can, in many cases, assign the delayed aircraft to another formation. The present framework cannot accommodate such a dynamic scheduling without modifications. However, since we do not propagate delays in multi-stage flights, the true robustness of formation flight in the context of real world operations is still uncertain and warrants further research. 

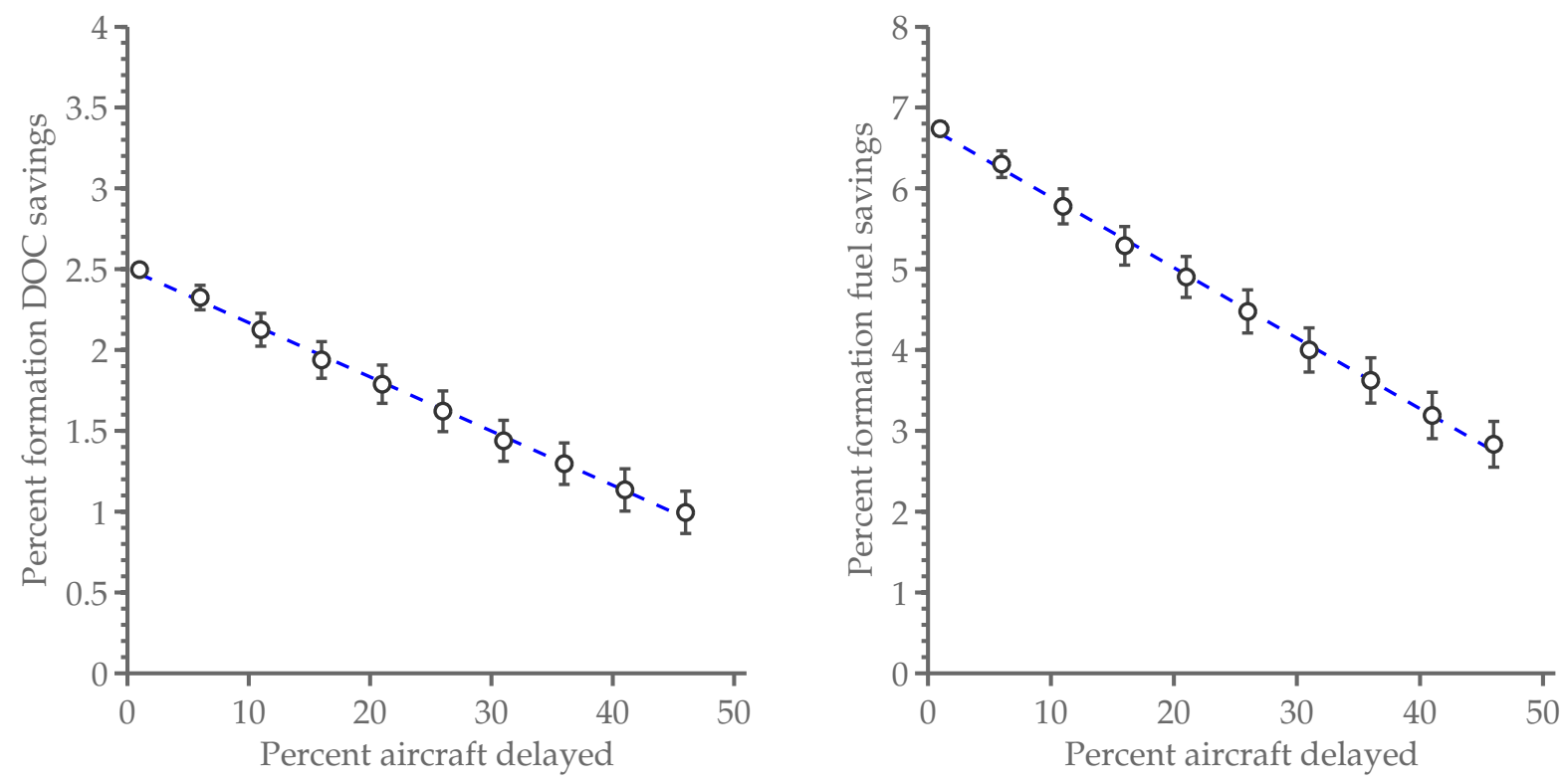

Figure 19. The impact of flight delay (modeled as a random percentage of flights that cannot join in formations) on the performance of the Star Alliance schedule. Lines correspond to linear fits of the average fuel and cost savings.

\section{Conclusion}

In this paper, we demonstrate a bi-level decomposition scheme to optimize airline schedules for extended formation flight. The design framework is unique in its combination of aircraft performance and aerodynamics with aircraft scheduling optimization. The scale of the formation flight routing problem motivates the application of heuristic filters to eliminate unlikely formations.

The results of design studies based on real-world flight schedules demonstrate that formation flight can produce tangible fuel and cost savings. A 31-flight South African Airlines long-haul schedule can reduce fuel burn by over $5.8 \%$, or reduce direct operating cost by $2.0 \%$ using formation flight. The savings increase when aircraft from multiple airlines fly in similar corridors. A 150-flight Star Alliance transatlantic schedule can expect to achieve a $7.7 \%$ reduction in fuel burn, or a $2.6 \%$ reduction in direct operating cost with formation flight. Finally, the results of a preliminary study demonstrate that the formation flight schedule can be effectively designed to cope with compressibility effects induced by wake vortices.

An important assumption that underpins the present analysis is that a trailing aircraft can accurately track the wake of the leading aircraft. This ability is both the cornerstone of formation drag reduction and the basis for safe formation flight. A substantial effort is still needed to understand the sensor and control system requirements for aircraft station keeping relative to wake vortices. However, some level of savings is possible even without wake tracking. Moreover, technologies to better characterize and track wakes in-fight are important in their own right for increasing traffic density and improving safety in heavily traveled flight corridors. The technical infrastructures for formation flight - airborne LIDAR and next generation air traffic control - may grow organically from other advances in commercial aviation. Opportunities exist therefore to incorporate formation flight requirements and priorities into related research areas to help offset the risk and cost associated with adopting this new operational paradigm.

For instance, formation flight considerations such as negotiating and planning the 4D trajectories for formation rendezvous and splitting should inform NEXTGEN requirements. Further paradigm changes have been discussed to reduce pilot and controller workload in the context of formation flight, such as having direct ATC communications with only the lead vehicle in the formation. 30

Another area of future work would be to explicitly account for both vertical and horizontal flight track restrictions. Clearly, actual formations would have to work within the constraints of current flight levels, which would have a similar impact on fuel burn as for solo routing where optimal continuous cruise-climb 
profiles cannot be flown. For horizontal flight track restrictions there are a couple of points worth noting. First, as formation flights make the most sense on longer routes, many of which are transoceanic, there is less of a need to plan routes through heavily constrained, or around restricted airspace, such as in Europe or the Eastern United States. Second, in heavily constrained airspace where current restrictions must remain intact, the formation flight mission optimization could be updated to include these constraints. This would be relatively straightforward to include, but could greatly increase the computational burden for each mission optimization. Other operational influences, such as routing along favorable winds or to avoid bad weather would also need to be incorporated in practical routing software.

Another unexplored issue is the effect of flight interruption on the efficiency and robustness of formation flight. In the present study, we require all aircraft in formation to carry sufficient fuel to fly the generally longer formation flight mission without any formation benefits. These contingency mission constraints improve robustness and safety, but lead to sub-optimal fuel burn as aircraft are burdened with excess fuel reserves.

Although a preliminary sensitivity study models the effect of single-stage delays on formation flight, we do not address what happens to a multi-legged formation flight schedule if a formation aircraft experiences delays or cancellation. Future work may have to incorporate more sophisticated cost objectives that are sensitive to flight disruption, cascading delays, random diversions, and passenger throughput.

Finally, the inclusion of larger and more complex formations with more than one set of rendezvous and separation points can increase the benefits of formation flight. However, the benefits from larger formations should be weighed against the diminishing returns in drag savings and increased coordination and station keeping complexity.

\section{Acknowledgement}

We gratefully acknowledge the support of Airbus SAS for this research.

\section{References}

\footnotetext{
${ }^{1}$ Wieselsberger, C., "Beitrag zur Erklärung des Winkelfluges einiger Zugvögel," Z. Flugtechnik $\&$ Motorluftschiffahrt, Vol. 5, 1914, pp. 225-229.

${ }^{2}$ Lissaman, P. B. S. and Shollenberger, C. A., "Formation Flight of Birds," Science, Vol. 168, No. 3934, 1970, pp. 1003, doi $10.1126 /$ science.168.3934.1003

${ }^{3}$ Hummel, D., "Aerodynamic Aspects of Formation Flight in Birds," Journal of Theoretical Biology, Vol. 104, No. 3, 1983, pp. 321-347.

${ }^{4}$ Weimerskirch, H., Martin, J., Clerquin, Y., Alexandre, P., and Jiraskova, S., "Energy Saving in Flight Formation," Nature, Vol. 413, No. 6857, 10 2001, pp. 697-698, doi 10.1038/35099670

${ }^{5}$ Blake, W. and Multhopp, D., "Design, Performance and Modeling Considerations for Close Formation Flight," AIAA Atmospheric Flight Mechanics Conference and Exhibit, AIAA-1998-4343, August 1998, pp. 476-486.

${ }^{6}$ Frazier, J. W. and Gopalarathnam, A., "Optimum Downwash Behind Wings in Formation Flight," Journal of Aircraft, Vol. 40, No. 4, Feb 2003, pp. 799-803, doi 10.2514/2.3162

${ }^{7}$ Wagner, E., Jacque, D., Blake, W., and Pachter, M., "Flight Test Results of Close Formation Flight for Fuel Savings," AIAA Atmospheric Flight Mechanics Conference and Exhibit, August 2002.

${ }^{8}$ Ray, R. J., Cobleigh, B. R., Vachon, M. J., and John, C. S., "Flight Test Techniques Used to Evaluate Performance Benefits During Formation Flight," TP-2002-210730, NASA, August 2002.

${ }^{9}$ Pahle, J., Berger, D., Venti, M. W., Faber, J. J., Duggan, C., and Cardinal, K., "A Preliminary Flight Investigation of Formation Flight for Drag Reduction on the C-17 Aircraft," AIAA Atmospheric Flight Mechanics Conference; Salt Lake City, UT, 2012.

${ }^{10}$ Ning, S. A., Flanzer, T., and Kroo, I., "Aerodynamic Performance of Extended Formation Flight," Journal of Aircraft, Vol. 48, No. 3, May 2011, pp. 855-865, doi $10.2514 / 1.54636$

${ }^{11}$ Bower, G. and Kroo, I., "Multi-Objective Aircraft Optimization for Minimum Cost and Emissions Over Specific Route Networks," 26th Congress of International Council of the Aeronautical Sciences, 2008.

${ }^{12}$ Bower, G., Flanzer, T., and Kroo, I., "Formation Geometries and Route Optimization for Commercial Formation Flight," 27th AIAA Applied Aerodynamics Conference, 2009.

${ }^{13}$ Ning, S. A. and Kroo, I., "Compressibility Effects of Extended Formation Flight," AIAA Applied Aerodynamics Conference, June 2011.

${ }^{14}$ Barnhart, C., Kniker, T., and Lohatepanont, M., "Itinerary-based Airline Fleet Assignment," Transportation Science, Vol. 36, No. 2, 2002, pp. 199-217.

${ }^{15}$ Lohatepanont, M. and Barnhart, C., "Airline Schedule Planning: Integrated Models and Algorithms for Schedule Design and Fleet Assignment," Transportation Science, Vol. 38, No. 1, 2004, pp. 19-32.

${ }^{16}$ Hane, C., Barnhart, C., Johnson, E., Marsten, R., Nemhauser, G., and Sigismondi, G., "The Fleet Assignment Problem: Solving a Large-scale Integer Program," Mathematical Programming, Vol. 70, No. 1, 1995, pp. 211-232.
} 
${ }^{17}$ Betz, A., "Behavior of Vortex Systems," TM-713, NACA, 1933.

${ }^{18}$ Delisi, D., Greene, G., Robins, R., Vicroy, D., and Wang, F., "Aircraft Wake Vortex Core Size Measurements," AIAA Applied Aerodynamics Conference, AIAA-2003-3811, June 2003.

${ }^{19}$ Holzäpfel, F., "Probabilistic Two-Phase Wake Vortex Decay and Transport Model," Journal of Aircraft, Vol. 40, No. 2, March 2003, pp. 323-331, doi $10.2514 / 2.3096$

${ }^{20}$ King, R. M. and Gopalarathnam, A., "Ideal Aerodynamics of Ground Effect and Formation Flight," Journal of Aircraft, Vol. 42, No. 5, September 2005, pp. 1188-1199, doi 10.2514/1.10942

${ }^{21}$ Ning, S. A., Aircraft Drag Reduction Through Extended Formation Flight, Ph.D. thesis, Stanford University, 2011.

${ }^{22}$ Rahm, S., Smalikho, I., and Köpp, F., "Characterization of Aircraft Wake Vortices by Airborne Coherent Doppler Lidar," Journal of Aircraft, Vol. 44, No. 3, 2007, pp. 799-805.

${ }^{23}$ Kroo, I., "An Interactive System for Aircraft Design and Optimization," Journal of Aircraft, Vol. 92-1190, 1992.

${ }^{24}$ McGeer, T. and Shevell, R., Method for Estimating the Compressibility Drag of an Airplane, Department of Aeronautics and Astronautics, Stanford University, 1983.

${ }^{25}$ Liebeck, R. and Center, L. R., Advanced Subsonic Airplane Design 85 Economic Studies, National Aeronautics and Space Administration, Lewis Research Center, 1995.

${ }^{26}$ Joslin, R., "Aircraft Laminar Flow Control," Annual Review of Fluid Mechanics, Vol. 30, No. 1, 1998, pp. 1-29.

${ }^{27}$ Green, J., "Laminar Flow Control: Back to the Future?" 38th AIAA Fluid Dynamics Conference and Exhibit, 2008.

${ }^{28}$ Allison, E. and Kroo, I., "Aircraft Conceptual Design with Laminar Flow," 27th International Congress of the Aeronautical Sciences, 2010.

29 "Transportation Statistics Annual Report," Tech. rep., U.S. Department of Transportation, 2010.

${ }^{30}$ Hill, A. and Hof, H., "Working Paper 395: FF-ICE Concept Elements for Flights which are not Operating According to ICAO Rules, and for Formation Flights," ICAO ATMRPP-WG-WHL10, Montreal, 2008. 